\title{
Integrated Bacterial and Fungal Diversity Analysis Reveals the Gut Microbial Alterations in Diarrheic Giraffes
}

\author{
Aoyun $\mathrm{Li}^{1,2+}$, Bingxian $\mathrm{Li}^{3+}$, Feiran $\mathrm{Li}^{2}$, Yuanyuan $\mathrm{He}^{2}$, Lei Wang ${ }^{4}$, \\ Muhammad Fakhar-e-Alam Kulyar', Huade Li ${ }^{5}$, Yuhang Fu' ${ }^{2}$, Huaisen $\mathrm{Zhu}^{2}$, \\ Yaping Wang ${ }^{2}$ and Xiong Jiang ${ }^{1 *}$
}

${ }^{1}$ Hubei Three Gorges Polytechnic, Yichang, China, ${ }^{2}$ College of Veterinary Medicine, Huazhong Agricultural University, Wuhan, China, ${ }^{3}$ College of Veterinary Medicine, South China Agricultural University, Guangzhou, China, ${ }^{4}$ Animal Husbandry Station of Bijie City, Bijie, China, ${ }^{5}$ Sichuan Academy of Grassland Science, Chengdu, China

\section{OPEN ACCESS}

Edited by:

Ernesto Perez-Rueda,

Universidad Nacional Autónoma de México, Mexico

Reviewed by: Sonia Dávila-Ramos, Universidad Autónoma del Estado de Morelos, Mexico Mario Alberto Martínez Núñez, Universidad Nacional Autónoma de México, Mexico

*Correspondence: Xiong Jiang jiangx1025@sina.com

${ }^{\dagger}$ These authors have contributed equally to this work

Specialty section: This article was submitted to Microorganisms in Vertebrate Digestive Systems, a section of the journa Frontiers in Microbiology

Received: 19 May 2021

Accepted: 16 July 2021 Published: 12 August 2021

Citation:

Li A, Liu B, Li F, He Y, Wang L, Fakhar-e-Alam Kulyar M, Li H, Fu Y, Zhu H, Wang Y and Jiang $X(2021)$

Integrated Bacterial and Fungal Diversity Analysis Reveals the Gut

Microbial Alterations in Diarrheic Giraffes. Front. Microbiol. 12:712092.

doi: 10.3389/fmicb.2021.712092
Gut microbiota has been demonstrated to be associated with multiple gastrointestinal diseases, but information regarding the gut microbial alternations in diarrheic giraffe remains scarce. Here, 16S rDNA and ITS gene amplicon sequencing were conducted to investigate the gut microbial composition and variability in diarrheic giraffes. Results demonstrated that Firmicutes and Proteobacteria were the most dominant phyla in the gut bacterial community, whereas Ascomycota and Basidiomycota were observed to be predominant in the gut fungal community regardless of health status. However, the species and relative abundance of preponderant bacterial and fungal genera in healthy and diarrheic giraffes were different. In contrast to the relatively stabilized gut fungal community, gut bacterial community displayed a significant decrease in the alpha diversity, accompanied by distinct changes in taxonomic compositions. Bacterial taxonomic analysis revealed that the relative abundances of eight phyla and 12 genera obviously increased, whereas the relative abundances of two phyla and eight genera dramatically decreased during diarrhea. Moreover, the relative richness of five fungal genera significantly increased, whereas the relative richness of seven fungal genera significantly declined in diarrheic giraffes. Taken together, this study demonstrated that diarrhea could cause significant alternations in the gut microbial composition of giraffes, and the changes in the gut bacterial community were more significant than those in the gut fungal community. Additionally, investigating the gut microbial characteristics of giraffes in different health states is beneficial to provide a theoretical basis for establishing a prevention and treatment system for diarrhea from the gut microbial perspective.

Keywords: gut microbiota, diarrhea, 16S rDNA, ITS, giraffe

\section{INTRODUCTION}

Ruminant intestines are colonized by trillions of microbes which play crucial roles in immune system maturation, intestinal epithelial mucosal barrier maintenance, metabolism, and nutrient absorption (Garrett et al., 2010; Arumugam et al., 2011; Tremaroli and Backhed, 2012; Backhed et al., 2015). Statistical analysis indicates that approximately $98 \%$ of intestinal microbes 
are bacteria, whereas the remaining contains fungi (about $0.1 \%$ ), protozoa, and viruses (Garrett et al., 2010; Tremaroli and Backhed, 2012; Backhed et al., 2015). These microbes display a symbiotic relationship with the host through complicated networks of interactions and crosstalk with each other (Aziz et al., 2013; Guo et al., 2015). Remarkably, some potentially pathogenic microorganisms may also inhabit as parts of normal gut microbiota but may take opportunity to cause disease, e.g., gut microbial alteration and immune dysregulation of host (Aziz et al., 2013; Guo et al., 2015). Gut microbial community evolves with host and has become a vital organ affecting its health (Cryan and Dinan, 2012; Zhang et al., 2020). Several studies provided supporting evidence that shifts in the gut microbial composition that could extend its adverse effects beyond the gastrointestinal system and affect the functions of extra-intestinal organs, such as the brain and liver (Zhang et al., 2020). Gut microbial community has been demonstrated to be involved in the development of multiple diseases such as colorectal cancer, diabetes, obesity, and dyspepsia (Delzenne et al., 2011; Musso et al., 2011; Ambalam et al., 2016). Although gut bacterial importance has been well demonstrated by numerous studies, analyses regarding the relationship between gut fungal communities and host health have been insufficient to date.

Diarrhea is one of the leading causes of decreased productivity and death in ruminants that has been considered a vital factor impeding animal husbandry development in many countries (Musso et al., 2011; Ambalam et al., 2016). Early investigations revealed that diarrhea was present in almost all ruminants and especially epidemic in neonatal goat, sheep, cattle, and yak with immature gastrointestinal tract, which caused approximately half of all ruminant deaths ( $\mathrm{Li}$ et al., 2018; Bu et al., 2020; Xue et al., 2020). Previous research has indicated that some intestinal microbes including bacteria and fungi of ruminants alternate between preponderant and weak populations accompanied by diarrheic symptoms (Yang et al., 2017; Wang et al., 2018; Wang et al., 2019a). Therefore, some inevitable associations may be present between alternations in gut microbial community and diarrhea, but its specific connections and laws remain to be determined. Previously, numerous studies were performed on pathogenic bacteria, parasite, and virus to reveal the cause of ruminant diarrhea (Gallardo et al., 2020; He et al., 2020). Recent studies on gut microbiota of goat, yak, and mice have provided evidence that gut microbial dysbiosis may be one of the reasons of diarrhea (Han et al., 2017; Shao et al., 2020).

Metagenomic analysis based on high-throughput sequencing technology is an efficient tool for characterizing gut microbial composition and diversity differences after suffering certain diseases and has made possible in systematically investigating the relationship between host diseases and gut microbiota (Han et al., 2017; Shao et al., 2020). Moreover, in-depth comparison and analysis of obtained gut microbial information contributed to further understand the mechanisms causing ill health and develop the strategies to minimize the collateral damage (Han et al., 2017; Shao et al., 2020). Giraffe (Giraffa camelopardalis) mainly inhabiting the African continent is the tallest terrestrial ruminant in the world and displays a complicated gastrointestinal microbial ecosystem (Brown et al., 2007; AlZahal et al., 2016).
This species has been introduced into zoos around the world but showed a high diarrheic rate due to the alterations in habitat and survival environment (Brown et al., 2007; Mulherin et al., 2008; AlZahal et al., 2016). However, to date, the relationship between gut microbial composition and diversity and diarrhea in giraffes is still unclear. Taking advantage of this gap, we investigated the composition and variability of gut bacterial and fungal communities in the healthy and diarrheal giraffes by $16 \mathrm{~S}$ rDNA and ITS2 amplicon sequencing.

\section{MATERIALS AND METHODS}

\section{Sample Acquisition}

In this study, a total of twelve 4-year-old giraffes inhabiting in Yichang zoo (Yichang, China) were used for sample collection, including six healthy giraffes and six diarrheic populations. Additionally, the proportion of male and female in both groups was 1:1. These giraffes were maintained under the same conditions and possessed the same immune procedure. Prior to the sample acquisition, all the giraffes were observed and diagnosed by professional veterinarians to determine their health status and diarrheic populations without any medicinal treatment. Sufficient feed and water were provided ad libitum for all giraffes throughout the experimental period. One day prior to sample acquisition, healthy and diarrheic giraffes were placed in separate pens to prevent infection and sample contamination. Six individual fresh fecal samples were collected from each giraffe via using sampler the following morning. Subsequently, the obtained rectal feces were resampled from the intermediate portion to minimize pollution through bedding and flooring. Finally, all the samples were placed immediately in sterile plastic containers, snap-frozen using liquid nitrogen and stored at $-80^{\circ} \mathrm{C}$ for subsequent DNA extraction.

\section{S rDNA and ITS Gene Amplification and Sequencing}

The bacterial and fungal genomic DNA was extracted from feces of giraffes using QIAamp DNA Mini Kit (QIAGEN, Hilden, Germany) following suggested instructions of manufacturer. The total microbial DNA was quantified via utilizing UV-Vis spectrophotometer (NanoDrop 2000, United States), and DNA integrity was evaluated by $0.8 \%$ agarose gel electrophoresis. Bacterial 16S rDNA (338F: ACTCCTACGGGAGGCAGCA and 806R: GGACTACHVGGGTWTCTAAT) and fungal ITS gene primers (ITS5F: GGAAG TAAAAGTCGTAACAAGG and ITS2R: GCTGCGTTCTTCATCGA TGC) with special barcodes were synthesized on the basis of the conservative regions to amplify the V3/V4 and ITS2 regions, respectively. To ensure the accuracy of the results, PCR amplification was conducted in triplicates under the same conditions. The agarose gel electrophoresis (2\%) and AxyPrep DNA Gel Extraction Kit (Axygen, Union City, CA, United States) was employed to evaluate PCR amplification product and recycle target fragment, respectively. PCR-recycled products were performed fluorescent quantitation on Microplate reader (BioTek, FLx800) based on the original electrophoretic results. According to the fluorescence 
quantitative results and the requirements of sequencing quantity, the samples were mixed in corresponding proportions. The obtained PCR products were used to construct the sequencing library via using TruSeq Nano DNA LT Library Prep Kit (Illumina, San Diego, CA, United States). Meanwhile, the 2\% agarose gel electrophoresis was used for the final fragment selection and purifying the library. The quality inspection and fluorescence quantification of the sequencing libraries were performed before sequencing. The qualified libraries were diluted and then mixed in corresponding proportions based on the required sequencing quantity. The final libraries were subjected to high-throughput sequencing via MiSeq sequencing machine.

\section{Bioinformatics and Statistical Analysis}

The original data produced from high-throughput sequencing were performed by quality screening using QIIME software (Qiime1.9.1) to achieve reliable results in the subsequent bioinformatics analysis. The interrogative sequences including short sequences ( $<200 \mathrm{bp}$ ), mismatched primers, and chimera were discarded utilizing QIIME software (Qiime1.9.1), and the qualified sequences were assigned to the corresponding individuals according to the information of primer and barcode. The obtained sequences were OTU partitioned and clustered based on $97 \%$ similarity, and the representative sequences were conducted through classification identification and phylogenetic analysis. The gut microbial alpha diversity was calculated according to the relative abundance distribution of OTU in each sample. Beta diversity was calculated based on weighted and unweighted UniFrac distance to evaluate the difference and similarity in different samples and groups. Simultaneously, the rarefaction curve of each sample was generated to assess the sequencing depth. Statistical analysis of data was performed using $\mathrm{R}$ (v3.0.3) and GraphPad Prism (version 7.0c). $p$-values $<0.05$ were considered statistically significant, and the values were presented as means $\pm \mathrm{SD}$.

\section{RESULTS}

\section{Data Acquisition and Analysis}

In the present microbiome investigation, 12 fecal samples from healthy and diarrheic giraffes were subjected to amplicon sequencing and a total of 958,862 $(\mathrm{CG}=479,660, \mathrm{DG}=479,202)$ and 960,350 ( $\mathrm{CG}=480,389, \mathrm{DG}=479,961)$ original sequences were acquired from the V3/4 and ITS2 regions, respectively (Table 1). After sequence filtering, 1,795,319 high-quality reads were totally acquired from all the samples, with an average of 70,620 (ranging from 68,577 to 72,501) and 78,989 (varying from 78,434 to 79,841 ) reads from bacterial and fungal populations per sample, respectively (Table 2). Following taxonomic assignment, a total of 936 bacterial OTUs and 744 fungal OTUs were recognized based on $97 \%$ nucleotide-sequence similarity (Figures 1A,B,G,H). Moreover, we also observed 817 and 364 core OTUs in the bacterial and fungal communities, which accounted for approximately $87.29 \%$ and $48.92 \%$ of the total OTUs, respectively (Figures 1C,I). Both species accumulation and rarefaction curves in per sample were relatively flat and showed a tendency to saturate characteristics, indicating that nearly all the bacterial and fungal species were identified in fecal samples of giraffes (Figures 1D,E,J,K). Furthermore, the rank abundance curves for all samples were wide and fell gently, suggesting satisfactory evenness and abundance (Figures 1F,L).

\section{Shifts in Gut Microbial Diversities With the Effect of Diarrhea}

To further dissect the alternations of gut bacterial and fungal communities in diarrheic giraffe, the qualified sequences were aligned to estimate alpha and beta diversity indices. Gut bacterial and fungal alpha diversity could be characterized by sequencing depth (Good's coverage), species abundance (ACE), and species diversity (Shannon and Simpson). Good's coverage estimates varied from 99.79 to $99.96 \%$, suggesting that the majority of bacterial and fungal phenotypes presented in each sample were detected (Figures 2A,E). There was statistically significant differences in the gut bacterial ACE (834.53 \pm 56.67 versus $739.61 \pm 21.69, p=0.003)$ indices, whereas the Simpson index $(0.91 \pm 0.042$ versus $0.95 \pm 0.031, p=0.087)$ and Shannon $(5.53 \pm 0.65$ versus $6.16 \pm 0.59, p=0.11)$ indexes were not dramatically different between the CG and DG groups (Figures 2B-D). Intergroup analysis of alpha diversity intuitively showed that diarrhea observably decreased the gut bacterial abundance but had no effect on the bacterial diversity. Moreover, there was no significant difference in the gut fungal four $\alpha$ diversity indices in both groups, indicating a relatively stabilized gut fungal community in giraffes during the occurrence of diarrhea (Figures 2F-H). The principal coordinate analysis (PCoA) and unweighted pair-group method with arithmetic means (UPGMA) tree analysis were applied to assess the variabilities and similarity among intergroup and intragroup individuals. Both the weighted and unweighted PCoA plots showed that the individuals in the CG group were clustered closely and separated from the DG group, which was consistent with the results of UPGMA analysis, indicating that the gut bacterial and fungal principal compositions could be strongly influenced by the diarrhea (Figures 2I-N).

\section{Taxonomic Composition and Alteration of Gut Bacterial Community}

The relative proportion of preponderant taxa at the different taxonomical levels were detected through microbial taxon assignment, and significant alterations in the gut bacterial abundances and compositions were observed in both groups. At the phylum level, a total of 17 phyla were identified from the 12 samples, varying from 14 to 17 phyla per sample. Based on the phylum assignment result, Firmicutes $(55.21 \%$, $38.79 \%)$, Proteobacteria $(31.61 \%, 32.55 \%)$, Bacteroidetes $(8.19 \%$, $16.79 \%)$, and Actinobacteria $(2.17 \%, 6.46 \%)$ were the four most preponderant phyla in CG and DG groups, which accounted for approximately $95 \%$ of the total taxonomic groups identified in all samples (Figure 3A). Other phyla such as Verrucomicrobia $(0.22 \%, 0.55 \%)$, Tenericutes $(0.33 \%, 0.34 \%)$, Kiritimatiellaeota $(0.02 \%, 0.33 \%)$, and Synergistetes $(0.02 \%, 0.23 \%)$ in both groups were identified in low abundance. To further investigate the 
TABLE 1 | The bacterial sequence information of each sample.

\begin{tabular}{|c|c|c|c|c|c|c|}
\hline Sample & Raw reads & Clean reads & Effective reads & AvgLen (bp) & GC (\%) & Effective (\%) \\
\hline CG1 & 79,898 & 79,389 & 70,069 & 422 & 53.25 & 87.7 \\
\hline CG2 & 79,747 & 79,277 & 68,993 & 422 & 53.65 & 86.51 \\
\hline CG3 & 79,912 & 79,401 & 68,577 & 420 & 53.05 & 85.82 \\
\hline CG4 & 80,066 & 79,565 & 70,631 & 418 & 53.71 & 88.22 \\
\hline CG5 & 79,912 & 79,286 & 70,064 & 424 & 53.28 & 87.68 \\
\hline CG6 & 80,125 & 79,660 & 69,945 & 420 & 52.79 & 87.29 \\
\hline DG1 & 79,811 & 79,378 & 69,500 & 421 & 54.07 & 87.08 \\
\hline DG2 & 79,677 & 79,196 & 71,013 & 419 & 54.38 & 89.13 \\
\hline DG3 & 79,983 & 79,528 & 72,110 & 418 & 54.42 & 90.16 \\
\hline DG4 & 79,699 & 79,288 & 72,501 & 419 & 54.2 & 90.97 \\
\hline DG5 & 80,049 & 79,603 & 72,133 & 419 & 54.21 & 90.11 \\
\hline DG6 & 79,983 & 79,497 & 71,908 & 421 & 54.6 & 89.90 \\
\hline
\end{tabular}

TABLE 2 | The fungal sequence information of each sample.

\begin{tabular}{|c|c|c|c|c|c|c|}
\hline Sample & Raw reads & Clean reads & Effective reads & AvgLen (bp) & GC (\%) & Effective (\%) \\
\hline CG1 & 80,228 & 79,749 & 79,352 & 228 & 44.76 & 98.91 \\
\hline CG2 & 80,062 & 79,593 & 79,557 & 251 & 50.72 & 99.37 \\
\hline CG3 & 79,851 & 79,325 & 79,310 & 230 & 44.32 & 99.32 \\
\hline CG4 & 79,832 & 78,434 & 74,738 & 255 & 50.45 & 93.62 \\
\hline CG5 & 80,134 & 79,617 & 79,494 & 237 & 46.7 & 99.20 \\
\hline CG6 & 80,282 & 79,841 & 79,836 & 231 & 46.01 & 99.44 \\
\hline DG1 & 80,110 & 79,630 & 78,957 & 237 & 47.03 & 98.56 \\
\hline DG2 & 79,829 & 79,269 & 79,242 & 251 & 49.41 & 99.26 \\
\hline DG3 & 79,679 & 79,155 & 79,060 & 243 & 47.78 & 99.22 \\
\hline DG4 & 80,093 & 79,569 & 79,500 & 246 & 47.59 & 99.26 \\
\hline DG5 & 79,911 & 79,085 & 79,057 & 256 & 42.15 & 98.93 \\
\hline DG6 & 80,339 & 79,787 & 79,772 & 240 & 48.26 & 99.29 \\
\hline
\end{tabular}

effect of diarrhea on taxonomic compositions, 194 genera were detected from the gut microbiota of giraffes. Among these genera identified, Escherichia-Shigella (16.98\%) was the most predominant bacterial genus in the CG group, followed by Acinetobacter (13.11\%) and Solibacillus (11.63\%) (Figure 3B). However, Escherichia-Shigella (12.41\%), Acinetobacter (7.71\%), and Comamonas (11.36\%) were abundantly present in the DG group, accounting for over $31 \%$ of overall bacterial composition. The genus-level cluster analysis employing heatmap revealed the distribution of the bacterial genus in different samples and indicated the influence of diarrhea on the bacterial genus-level compositions (Figure 3E).

To further dissect the shifts in taxonomic compositions of giraffes in different health states, Metastats analysis was performed for different classification levels. A comparison of the DG and CG groups revealed a significant increase $(p<0.05$ or $p<0.01)$ in the abundance of Synergistetes, Fibrobacteres, Patescibacteria, Chloroflexi, Kiritimatiellaeota, Actinobacteria, Bacteroidetes, and Verrucomicrobia, as well as a distinct decrease $(p<0.01)$ in the abundance of Spirochaetes and Firmicutes (Figure 4A). At the genus level, 20 genera were totally identified to be dramatically different between both groups. Of these discriminatory taxa, the relative abundances of 12 bacterial genera significantly increased, whereas the relative abundances of eight bacterial genera dramatically decreased under the influence of diarrhea (Figure 4B). Considering that this discriminant analysis cannot distinguish the dominant taxon, linear discriminant analysis effect size (LEfSe) analysis coupled with linear discriminant analysis (LDA) was conducted to identify the specific bacteria related to diarrhea (Figure 5). Results revealed that at the phylum level, the abundance of Actinobacteria and Patescibacteria in the DG group were significantly preponderant than the CG group, while the Firmicutes was lower. At the genus level, the DG group was significantly enriched for Comamonas, Prevotellaceae_UCG_001, Succiniclasticum, and Corynebacterium_1, whereas the CG group showed a significantly higher abundance of Clostridium_sensu_stricto_1, Lysinibacillus, Bacillus, Romboutsia, Psychrobacillus, and Solibacillus.

\section{Significant Alterations in Gut Fungal Taxonomic Compositions in Diarrheic Giraffe}

There were nine phyla and 262 genera identified in the gut fungal communities of all giraffes using RDP classifier. The top 10 phyla and 10 genera of gut fungal community in both groups are presented in Figures 3C,D. The phyla Ascomycota $(\mathrm{CG}=70.63 \%, \mathrm{DG}=72.63 \%)$ and Basidiomycota $(\mathrm{CG}=25.84 \%$, 
A

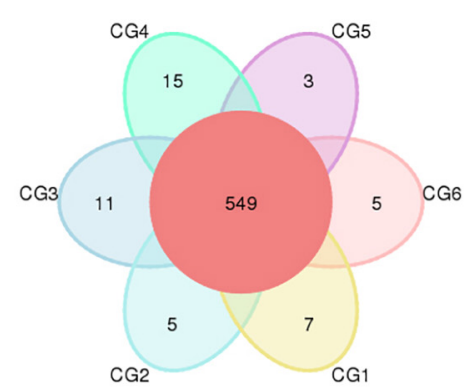

D

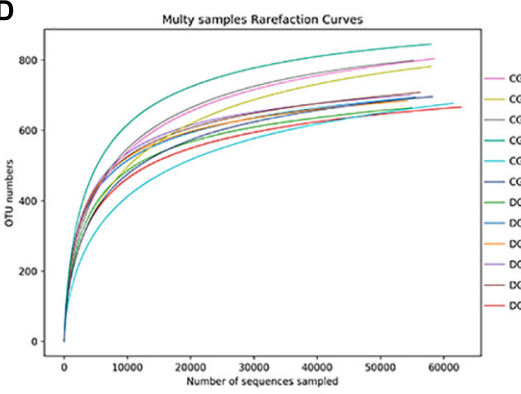

G

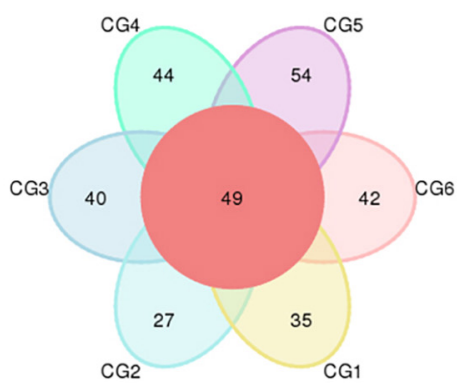

B

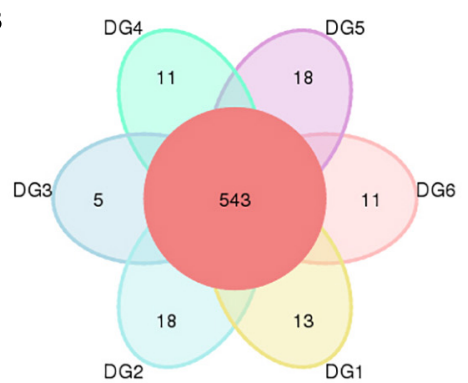

E

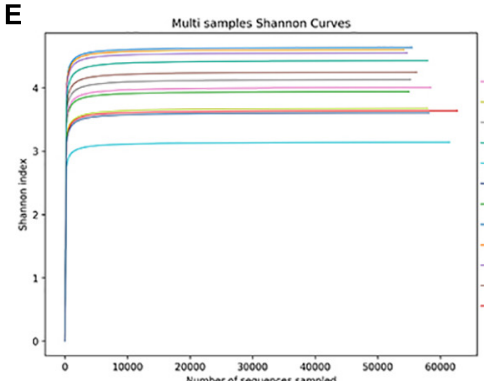

H
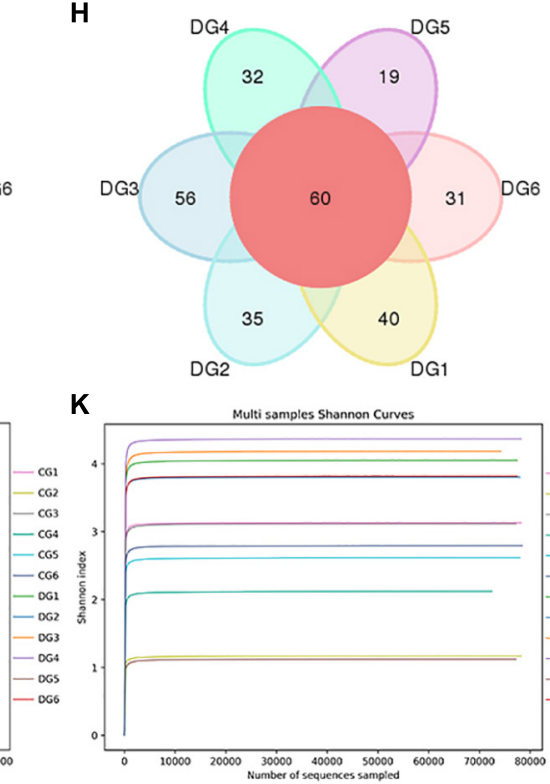

K

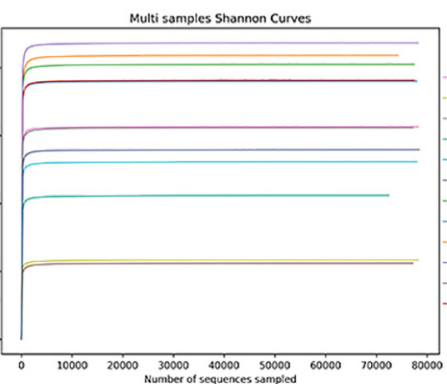

C

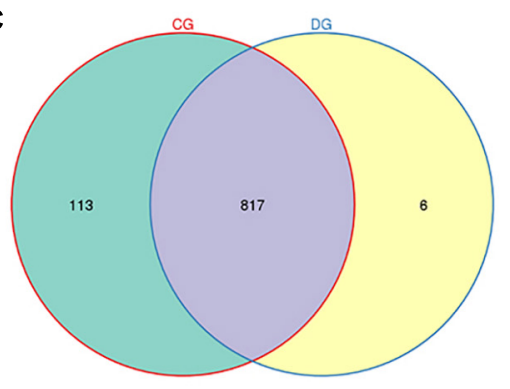

$\mathbf{F}$

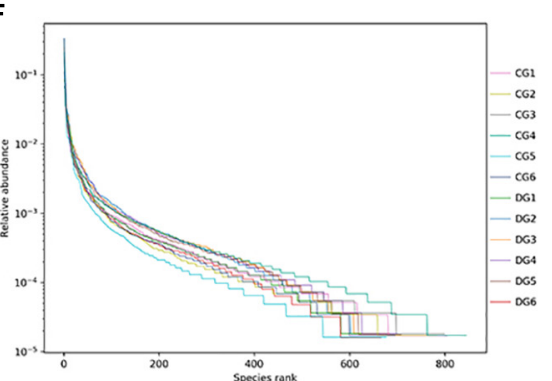

I

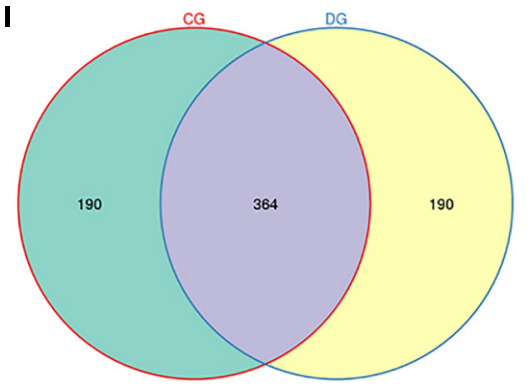

L

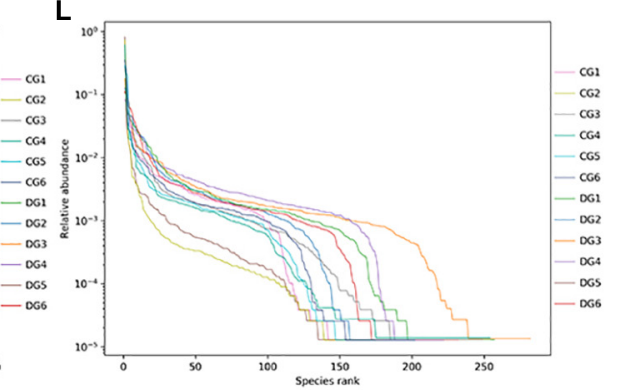

J

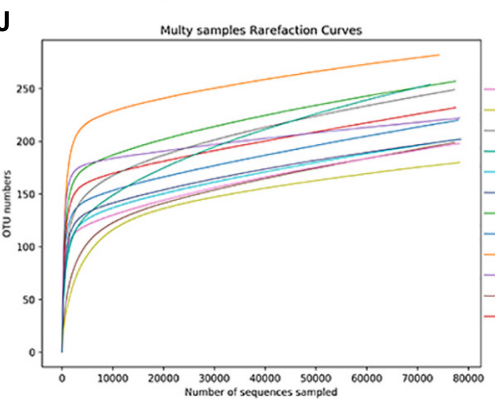

FIGURE 1 | Gut bacterial and fungal OTU distribution and feasibility analysis. (A-C) Venn diagrams for gut bacterial OTU distribution. (D-F) Bacterial rarefaction and rank abundance curves were used for assessing the quality of sequencing including depth, abundance, and evenness. (G-I) Venn diagrams for gut fungal OTU distribution. (J-L) Fungal rarefaction and rank abundance curves were used to evaluate the quality of sequencing including depth, abundance, and evenness. Each colored curve displayed in the figures represents a sample.

$\mathrm{DG}=18.01 \%$ ) were the most dominant fungi in giraffes regardless of health status, which together consisted of over 90\% of the fungal composition (Figure 3C). Other fungal phyla such as Chytridiomycota $(0.28 \%, 1.07 \%)$, Rozellomycota $(0.16 \%$, $0.25 \%)$, Neocallimastigomycota $(0.11 \%, 0.18 \%)$, Olpidiomycota $(0.01 \%, 0.06 \%)$, and Glomeromycota $(0.02 \%, 0.05 \%)$ in the CG and DG groups were represented with a lower abundance. At the genus level, the dominant fungal genera observed in the CG group were Trichosporon (20.76\%), Aspergillus (2.95\%), and Thelebolus (9.44\%), whereas Ascobolus (14.33\%), Aspergillus
(13.81\%), and Microascus (12.14\%) were enriched in the DG group (Figure 3D). The heatmap showed a higher similarity of the individuals within group than that among groups and revealed the alternations in fungal genus-level compositions under the effect of diarrhea (Figure 3F).

Using Metastats analysis to investigate the fungal genus-level taxonomic compositions in both groups, we observed that the relative abundances of five genera (Microascus, Aspergillus, Rhodotorula, Scopulariopsis, and Roussoella) significantly increased, whereas the relative abundances of seven genera 
A

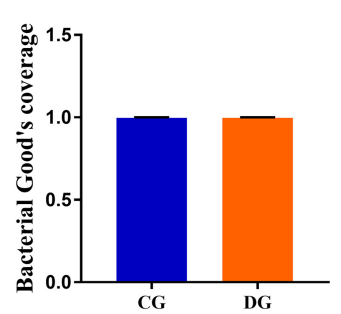

E

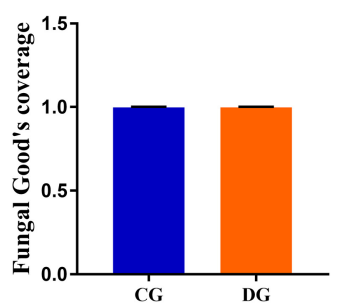

B

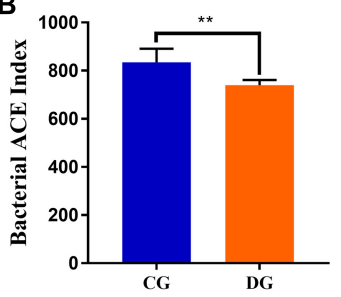

$\mathbf{F}$

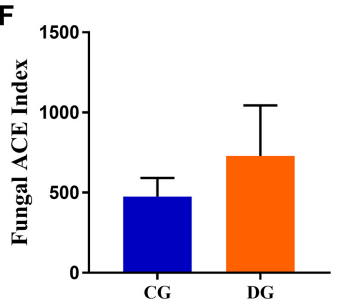

C

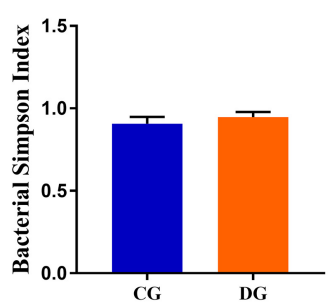

G

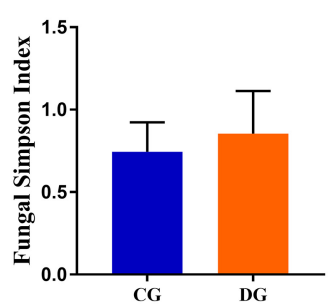

D

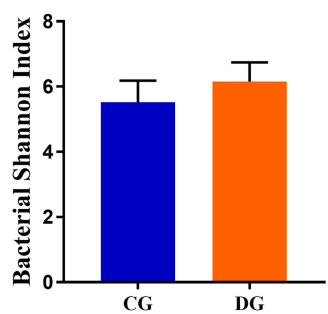

H

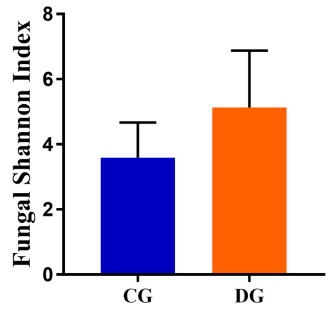

I

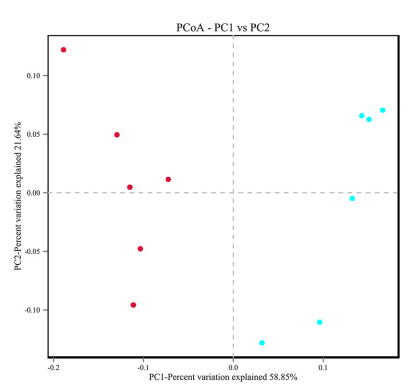

$\mathbf{L}$

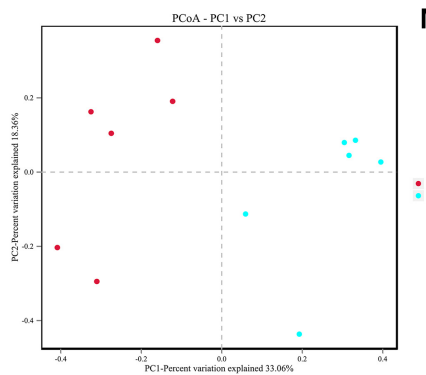

J

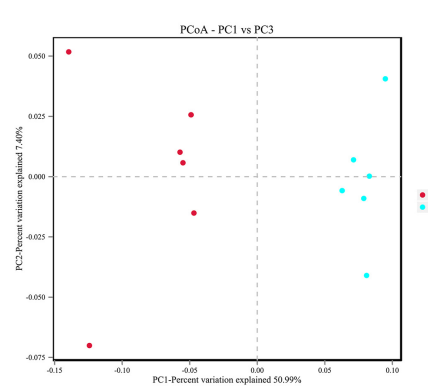

M

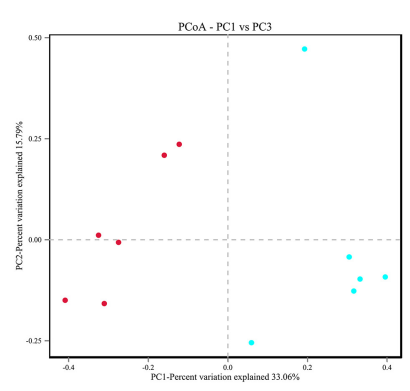

K

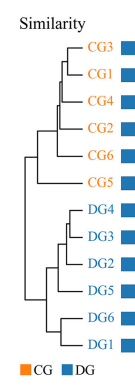

N

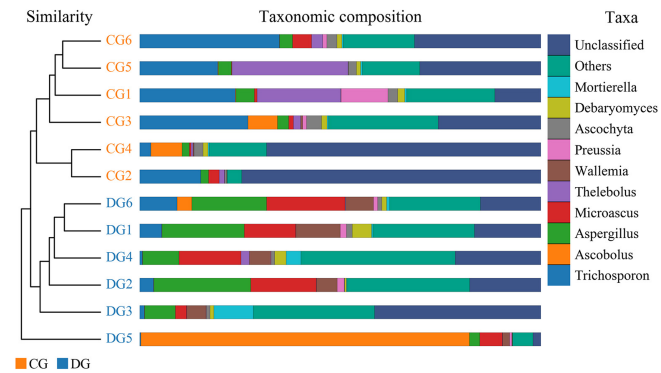

FIGURE 2 | The alternations of gut bacterial and fungal diversities during diarrhea. Gut bacterial and fungal alpha diversities can be evaluated by Good's coverage $(\mathbf{A}, \mathbf{E})$, ACE (B,F), Simpson (C,G), and Shannon (D,H). (I,J) Principal coordinate (PCoA) analysis based on the weighted and unweighted UniFrac distance of gut bacterial community. (L,M) Principal coordinate (PCoA) analysis of gut fungal community. (K,N) Gut bacterial and fungal clustering analysis based on unweighted pair-group method with arithmetic means (UPGMA). All of the data represent means $\pm \mathrm{SD}$. ${ }^{\star *} p<0.01$.

(Itersonilia, Neoascochyta, Mucor, Ustilago, Protostropharia, Cephaliophora, and Xeromyces) obviously decreased during diarrhea (Figure 6). Among them, two fungal genera (Itersonilia and Ustilago) even cannot be found in the gut fungal communities of diarrheic giraffes. LEfSe was applied to generate a cladogram to further investigate the variation in the fungal taxa composition (Figures 5C,D). Besides the above-mentioned significantly different funguses, we also observed that several funguses such as Wallemia and Cladorrhinum were the most dominant microbiota in the feces of patients in the DG group, whereas Botryotrichum, Thelebolus, and Trichosporon were significantly overrepresented in the CG group.

\section{Correlation Network Analysis}

Network analysis was conducted utilizing Python to illuminate linkages among different bacterial and fungal genera in gut microbiota (Figure 7). The bacterial network consisted of 80 nodes and 1,608 edges, whereas the fungal network was composed of 80 nodes and 266 edges. Results revealed that Ruminococcaceae_UCG-014 was positively associated with Rikenellaceae_RC9_gut_group (0.9231) and Alistipes (0.9371). Rikenellaceae_RC9_gut_group was positively associated with Alistipes (0.9790). Microascus was positively associated with Rhodotorula (0.8774). Aspergillus was positively associated with Rhodotorula (0.8194). 
A

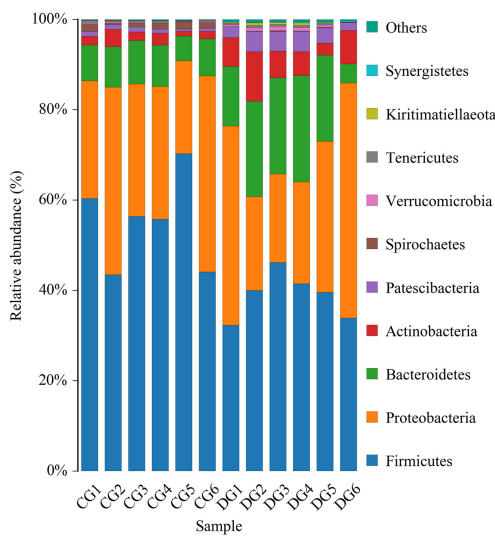

C

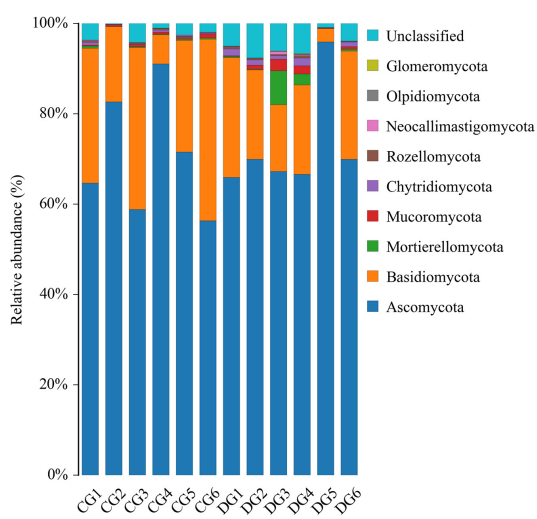

E

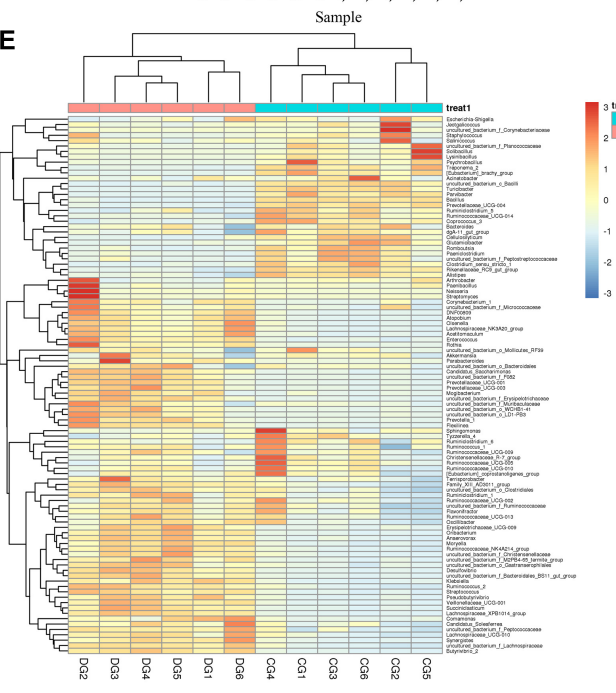

B

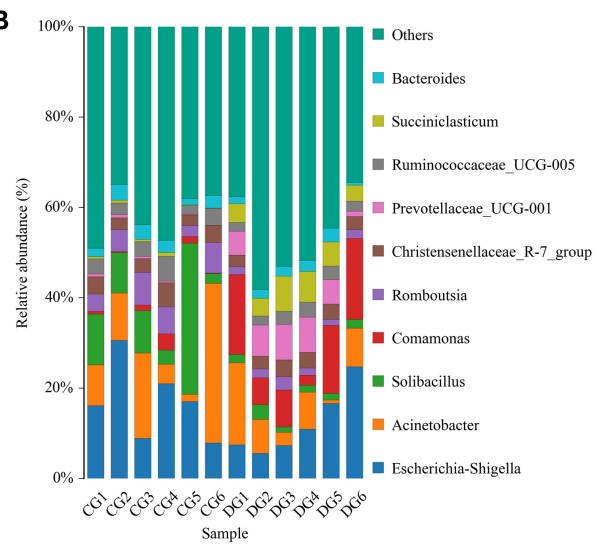

D

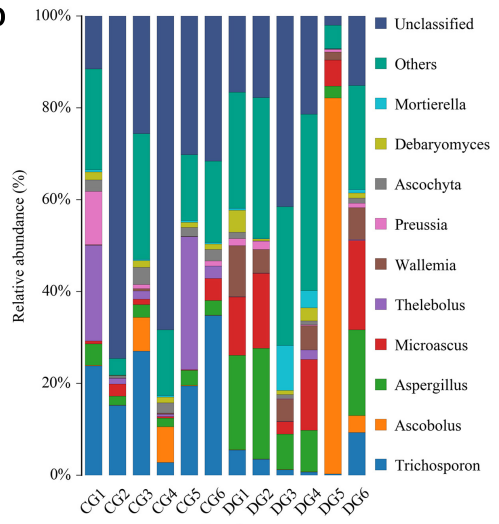

$\mathbf{F}$

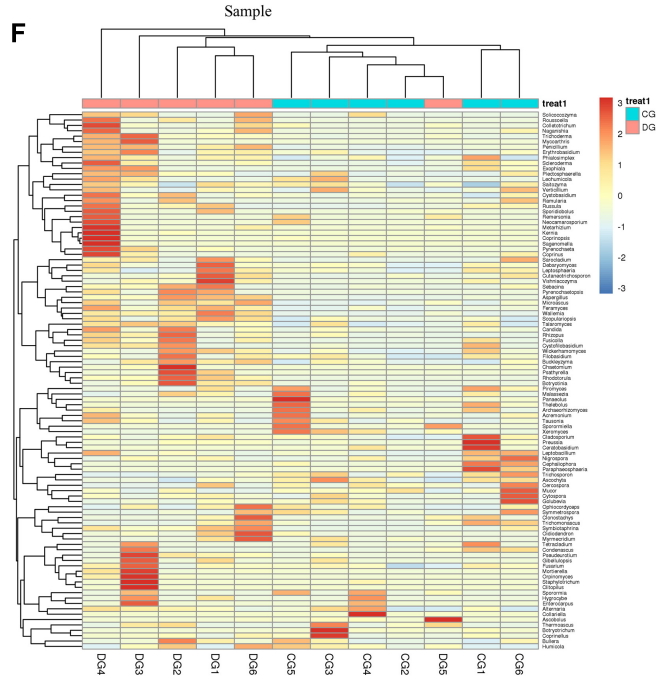

FIGURE 3 | The composition and relative abundance of the gut microbial community at the phylum and genus levels. (A,B) The gut bacterial relative abundance and composition of healthy and diarrheic giraffes at the phylum and genus levels. (C,D) The gut fungal relative abundance and composition of healthy and diarrheic giraffes at the phylum and genus levels. (E,F) Heatmap of the 50 most abundant gut bacterial and fungal genera in healthy and diarrheic giraffes.

\section{DISCUSSION}

Accumulating evidence demonstrated that the stable gut microbiota was a vital barrier for host against the invasion and colonization of foreign pathogens, whereas gut microbial alternations may be the driving or central factor of multiple diseases (Dumas et al., 2006; Wang et al., 2019b; Xiang et al., 2020). Thus, investigating the gut microbial composition and diversity may contribute to expand our understanding of disease etiology and provide convenient method to evaluate the health status of host. Gut microbiota is intimately involved in several other important activities including maintaining the 
A
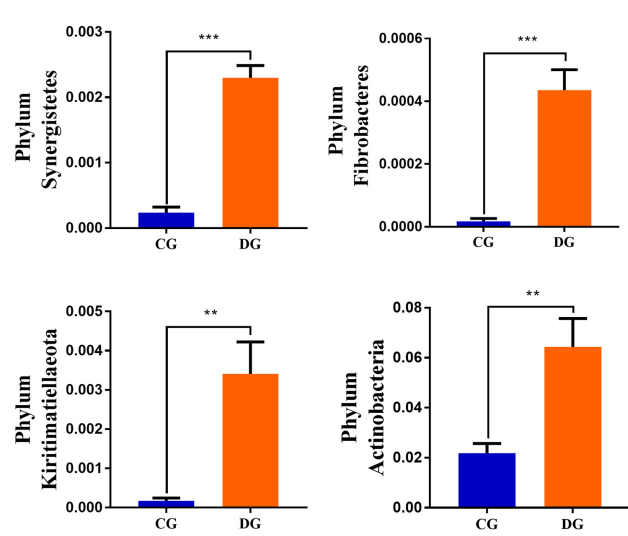

B
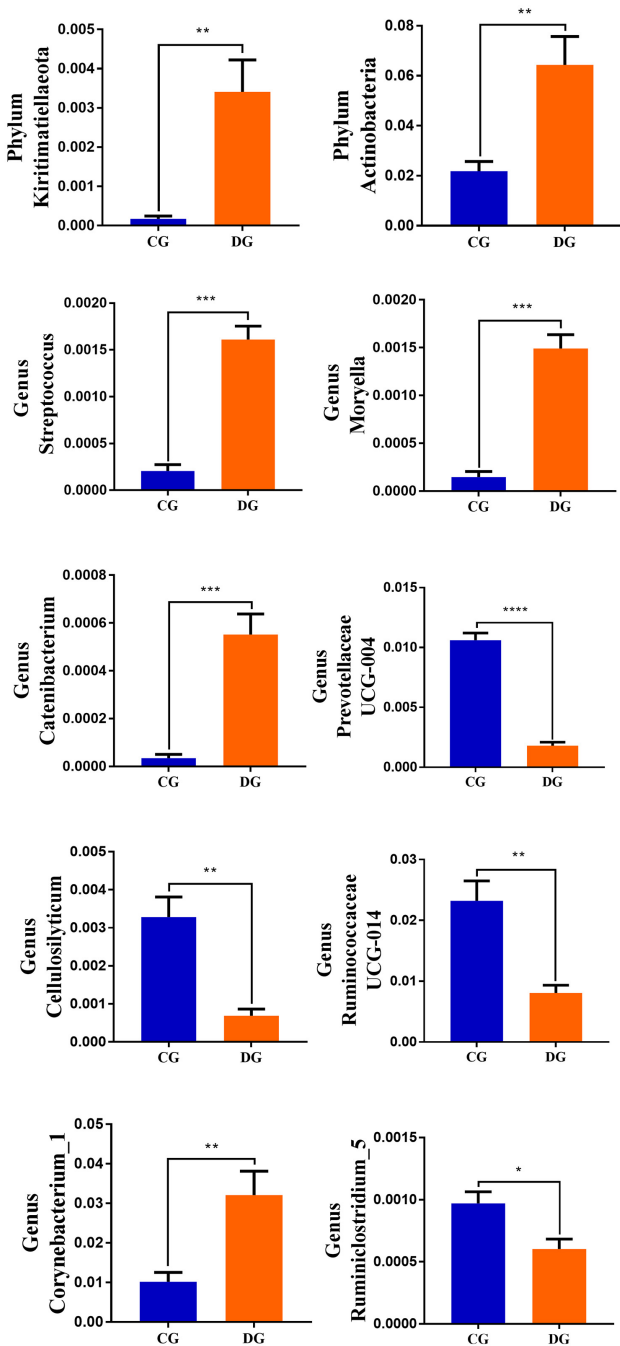
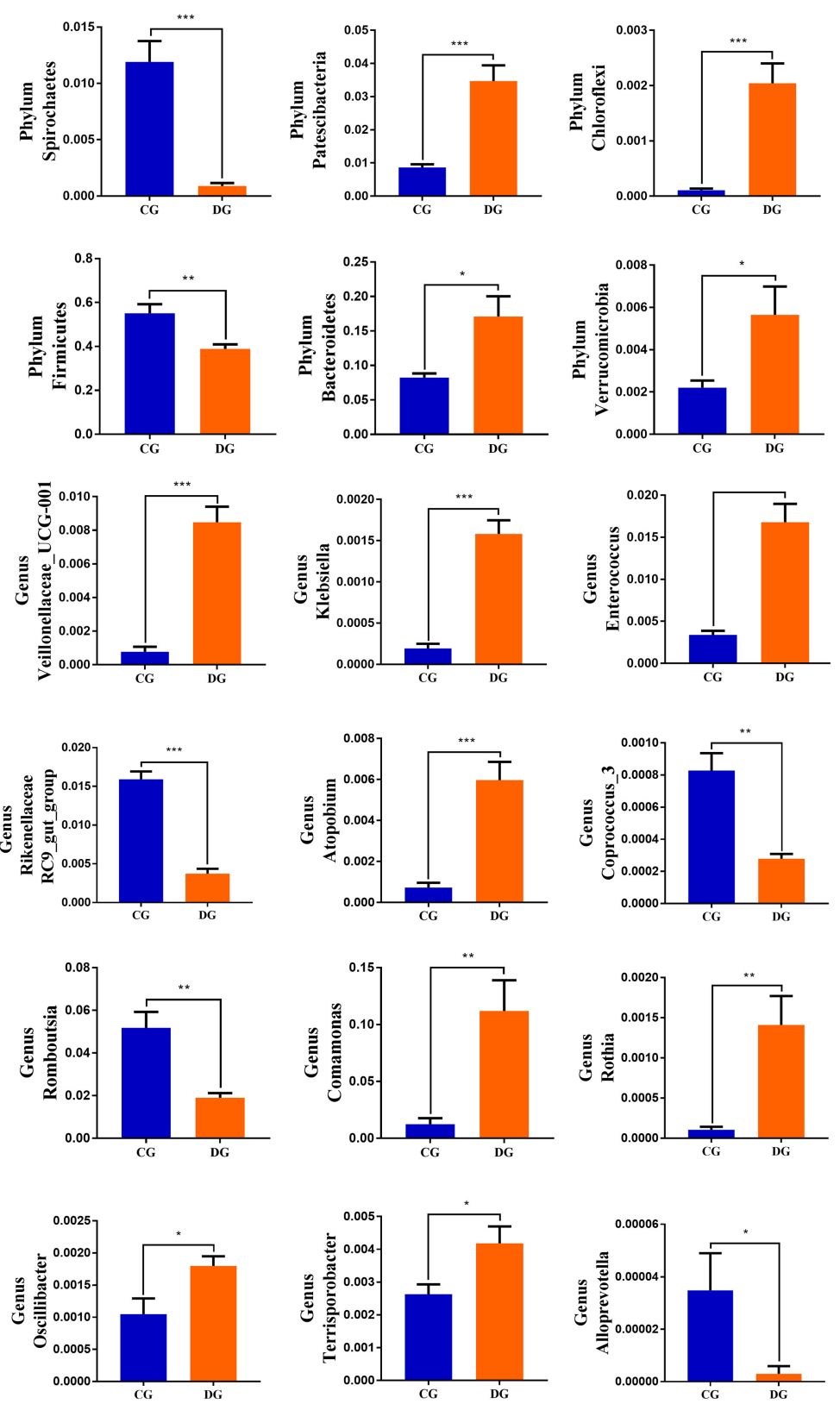

FIGURE 4 | Significant alternations in the gut bacterial abundance at the level of phylum (A) and genus (B) during diarrhea. All of the data represent means \pm SD. ${ }^{*} p<0.05 ;{ }^{* *} p<0.01 ;{ }^{* * *} p<0.001$ and ${ }^{* * * *} p<0.0001$

immunity, metabolism, and host health, which in turn depends on normal intestinal morphology (Li et al., 2021a). Diarrhea is one of the most common diseases of animals regardless of age and species, posing a great threat to public health, animal welfare, and animal husbandry. Moreover, the occurrence of diarrhea is inevitably related to gastrointestinal damage, indicating that the gut microbiota may be also altered. To date, research into the gut microbial composition and distribution in different health status has covered many species including goat, piglet, yak, and mice and demonstrated the significant variabilities of microbial community structure. However, studies regarding diarrheic influence on gut microbiota in giraffe have been insufficient to date. Taking advantage of this gap, we first characterized the variability of gut microbiota in diarrheic giraffes.

Generally, gut microbiota is dynamically diverse within limits and influenced by multiple factors such as species, age, sex, diet, and health status (Manichanh et al., 2012; Wang et al., 2020; Xiong et al., 2020; Zhu et al., 2020). Several studies have indicated that diarrhea was able to cause a significant decrease 


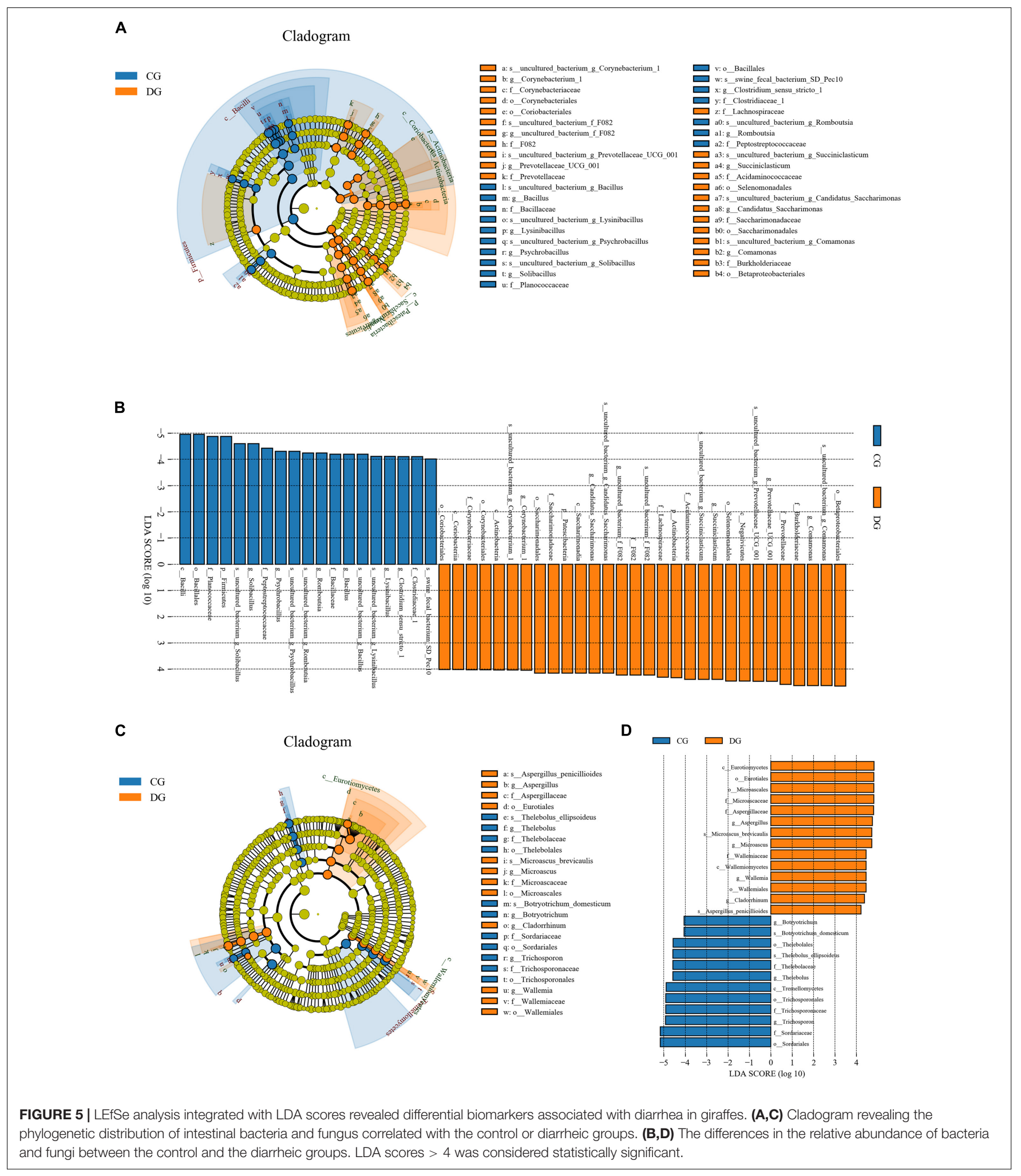

in the diversity of gut bacterial community as well as shifts in intestinal functions (Barash et al., 2017; He et al., 2020). Ma et al. (2020) revealed that the diversity of gut bacterial community in diarrheic rats was significantly decreased. Additionally, $\mathrm{He}$ et al. (2020) demonstrated a decreased alpha diversity of the gut bacterial community in diarrheic piglets. Consequently, diarrheic giraffes may be accompanied by significant changes in the gut microbial composition and structure. Considering 

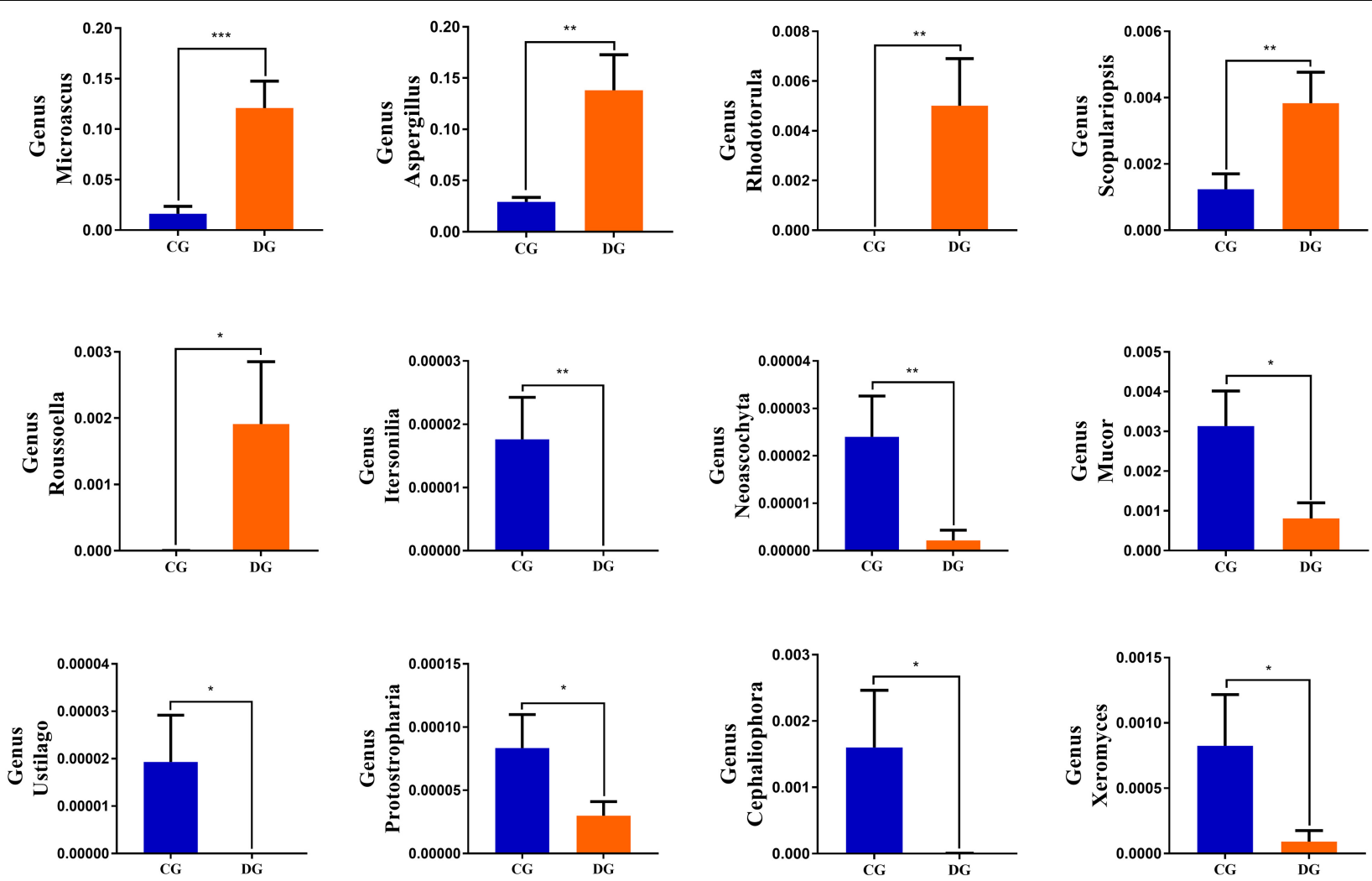

FIGURE 6 | Significant changes in the gut fungal abundance at the level of phylum and genus during diarrhea. ${ }^{\star} p<0.05 ;{ }^{\star \star} p<0.01$ and ${ }^{\star \star \star} p<0.001$ represent distinct difference between control and diarrheic groups.

the particularity of the species and the availability of samples, we selected fecal samples as the research object to assess the gut microbial composition and diversity. Consistent with previous studies, this study demonstrated a dramatically declined ACE index in the gut bacterial community of giraffe during diarrhea, implying the disorder of gut bacterial community. Several previous studies have demonstrate that the abundance and diversity of gut microbiota was positively related to intestinal functions, thereby the intestine with higher microbial diversity and abundance favors the energy utilization and performing complicated physiological functions (Barash et al., 2017; He et al., 2020). Furthermore, imbalanced gut microbiota may result in increased intestinal permeability and decreased immunity, which may further promote the invasion by members of pathogenic bacteria and conditioned pathogen (Barash et al., 2017). Therefore, diarrheic giraffe may also be at risk of intestinal dysfunction and other complications under situation of decreased gut bacterial diversity. Gut fungi, as a key component of the microbial community, was also considered to be an important contributor to intestinal health and function (Barash et al., 2017). Previous research has indicated that the gut fungal diversity of patients with diarrhea-predominant irritable bowel syndrome was significantly different from that of healthy population (Shukla et al., 2015; Hong et al., 2020). Li et al. (2018) indicated that there was no significant difference in the diversity of the gut fungal community between diarrheic and healthy yaks. Moreover, Sangster et al. (2016) observed that the gut fungal community of diarrheic patients induced by Clostridium difficile infection did not change significantly as compared with healthy population. In this study, we also observed that the differences of gut fungal diversity between diarrheic and healthy giraffes were not significant. Therefore, we speculated that gut bacterial community played a major role in the occurrence of giraffe diarrhea, whereas gut fungi community was secondary. Moreover, PCoA also was performed to evaluate the differences in the main components of the gut bacterial and fungal communities between both groups. Results indicated that the individuals of control group were clustered together and separated from the diarrhea group, implying a distinct difference in the primary composition of the gut bacterial and fungal communities between both groups. This study indicated that despite of shared environment and diets, the giraffes showed significant alterations in the gut bacterial and fungal communities during diarrhea. Therefore, we speculated that diarrhea was the primary driving force for changes in gut bacterial and fungal communities of giraffes.

This study demonstrated that Firmicutes, Proteobacteria, and Bacteroidetes were the most dominant bacterial phyla, whereas Ascomycota and Basidiomycota were the most preponderant fungal phyla in gut microbial community of giraffes, regardless 


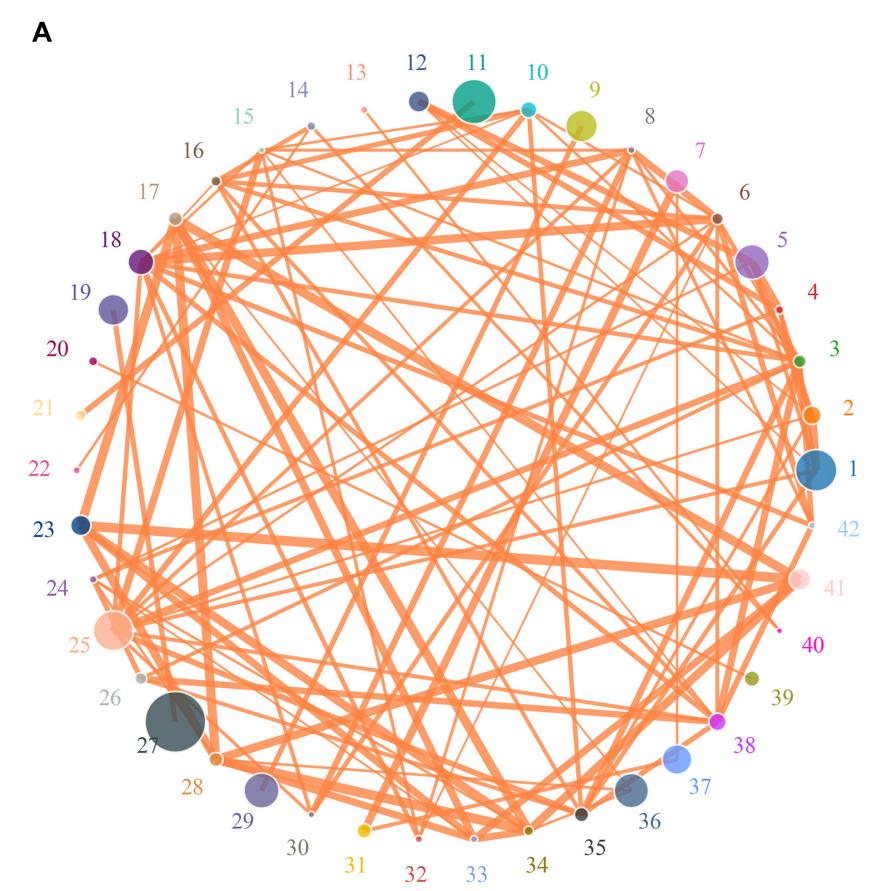

1: Prevotellaceae_UCG-001

2: Prevotellaceae_UCG-003

3: Lachnospiraceae_XPB1014_group

4: Flexilinea

5: Candidatus_Saccharimonas

6: Oribacterium

7: Rikenellaceae_RC9_gut_group

8: Moryella

9: Bacillus

10: Veillonellaceae_UCG-001

11: Romboutsia

12: Prevotella_1

13: Neisseria

14: Lachnospiraceae_UCG-010

15: Pseudobutyrivibrio

16: Butyrivibrio_2

17: Atopobium

18: Ruminococcaceae_NK4A214_group

19: Lysinibacillus

20: Oscillibacter

21: Cellulosilyticum
22: Synergistes

23: Lachnospiraceae_NK3A20_grc

24: Streptococcus

25: Succiniclasticum

26: Ruminococcus_2

27: Solibacillus

28: DNF00809

29: Psychrobacillus

30: Erysipelotrichaceae_UCG-009

31: Alistipes

32: Parabacteroides

33: Rothia

34: Acetitomaculum

35: Desulfovibrio

36: Corynebacterium_1

37: Ruminococcaceae_UCG-014

38: Family_XIII_AD3011_group

39: Ruminococcaceae_UCG-013

40: Candidatus_Soleaferrea

41: Olsenella

42: Anaerovorax
B

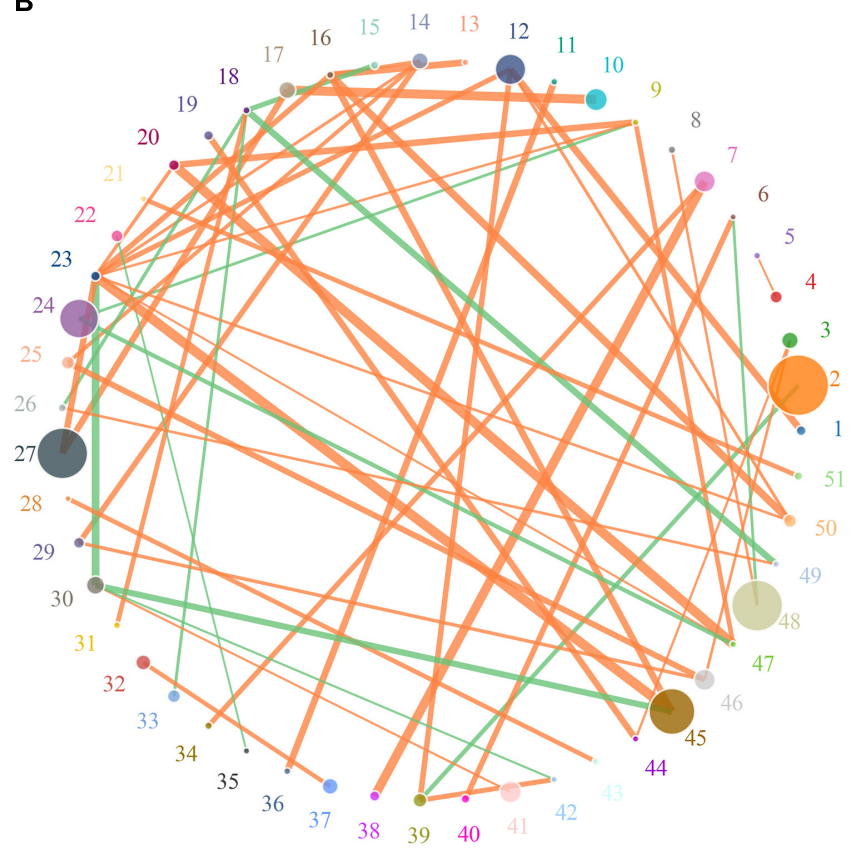

\begin{tabular}{|c|c|}
\hline 1: Scopulariopsis & 27: Aspergillus \\
\hline 2: Trichosporon & 28: Psathyrella \\
\hline 3: Fusarium & 29: Scleroderma \\
\hline 4: Coprinopsis & 30: Botryotrichum \\
\hline 5: Leohumicola & 31: Golubevia \\
\hline 6: Pyrenochaetopsis & 32: Coprinellus \\
\hline 7: Debaryomyces & 33: Candida \\
\hline 8: Collariella & 34: Verticillium \\
\hline 9: Pseudeurotium & 35: Archaeorhizomyces \\
\hline 10: Preussia & 36: Colletotrichum \\
\hline 11: Oidiodendron & 37: Filobasidium \\
\hline 12: Wallemia & 38: Leptosphaeria \\
\hline 13: Naganishia & 39: Trichoderma \\
\hline 14: Penicillium & 40: Sebacina \\
\hline 15: Fusicolla & 41: Ascochyta \\
\hline 16: Symbiotaphrina & 42: Orpinomyces \\
\hline 17: Wickerhamomyces & $\mathrm{s} \bigcirc 43:$ Cytospora \\
\hline 18: Cercospora & 44: Exophiala \\
\hline 19: Mycoarthris & 45: Microascus \\
\hline 20: Buckleyzyma & 46: Mortierella \\
\hline 21: Tausonia & 47: Botryotinia \\
\hline 22: Chaetomium & 48: Ascobolus \\
\hline 23: Rhodotorula & 49: Cystofilobasidium \\
\hline 24: Thelebolus & 50: Feramyces \\
\hline 25: Erythrobasidium & 51: Saitozyma \\
\hline
\end{tabular}

FIGURE 7 | Correlation network reveals the correlation among the different bacterial (A) and fungal (B) genera. The circles with different colors indicate the name of bacterial and fungal genus, and their sizes represent relative abundance. The strength of the correlation between both genera is positively related to the thickness of the line. The green line between both genera represents a positive correlation, whereas the orange line indicates a negative correlation.

of health status. These bacterial and fungal phyla were also found to be abundantly presented in the gut microbiota of cattle, goat, and yak, which was shown to be the major characteristic of the gut microbial community in ruminants
(Li et al., 2018, 2021b). We further investigated the gut microbial variabilities of this common diarrhea of giraffe. The differences of specific bacteria and fungi can intuitively indicate the potential relationship between gut microbiota and 
diarrhea. Our results revealed distinct increases in the relative abundances of eight bacterial phyla (Synergistetes, Fibrobacteres, Patescibacteria, Chloroflexi, Kiritimatiellaeota, Actinobacteria, Bacteroidetes, and Verrucomicrobia) and significant declines in the relative abundances of two phyla (Spirochaetes and Firmicutes) during diarrhea. Firmicutes, consisting of a large amount of gram-positive bacteria, are responsible for converting complicated carbohydrates into reabsorbable substrates ( $\mathrm{Li}$ et al., 2018, 2021b). Moreover, most members of Firmicutes are considered beneficial bacteria, which helps in regulating systemic immune responses, maintaining intestinal environment and inhibiting opportunistic pathogens (Sun et al., 2016). Actinobacteria synergy with one partner or host can easily be transformed into pathogenic interactions with another (Miao and Davies, 2010). Consistent with our findings, Wang et al. (2018) also observed that the abundances of Actinobacteria and Verrucomicrobia in the gut microbiota of diarrheic goats were significantly increased. Synergistetes has been shown to cause periodontal disease (Vartoukian et al., 2009; McCracken and Nathalia, 2020).

Importantly, this study also found a high variation in some bacterial and fungal genera between healthy and diarrheic giraffes and this variation may play crucial roles in intestinal ecosystem and function. This study demonstrated significant increases in the relative abundances of 12 genera (Streptococcus, Moryella, Veillonellaceae_UCG-001, Klebsiella, Enterococcus, Catenibacterium, Atopobium, Comamonas, Rothia, Corynebacterium_1, Oscillibacter, and Terrisporobacter) as well as significant declines in the relative abundances of eight genera (Prevotellaceae_UCG-004, Rikenellaceae_RC9_gut_group, Coprococcus_3, Cellulosilyticum, Ruminococcaceae_UCG-014, Romboutsia, Ruminiclostridium_5, and Alloprevotella) with the effect of diarrhea. Prevotellaceae and Cellulosilyticum have been reported to be involved in the degradation and digestion of carbohydrate, pectin, and cellulose (Cai et al., 2010; De Filippo et al., 2010; O'Keefe et al., 2015). Rikenellaceae can degrade plant-derived polysaccharides and limit the development of colitis via stimulating T-regulatory cell differentiation (He et al., 2015; Peng et al., 2015; Dubin et al., 2016). Ruminococcaceae has long been regarded as a potential beneficial bacterium due to the positive regulation of the immune system and intestinal environment (Shang et al., 2016). Furthermore, Ruminococcaceae has been reported to be negatively associated with liver cirrhosis, non-alcoholic fatty liver, and increased intestinal permeability (Huang et al., 2015; Shang et al., 2016). Romboutsia, an obligate anaerobe, contains multiple metabolic capabilities associated with carbohydrate utilization and fermentation of single amino acids (Ricaboni et al., 2016; Xin et al., 2019). Alloprevotella can produce moderate amounts of acetate and succinate and decrease lifetime cardiovascular disease risk (Downes et al., 2013; Ricaboni et al., 2016; Xin et al., 2019). Some genera such as Coprococcus and Ruminiclostridium have been demonstrated to produce short-chain fatty acids, which is beneficial to regulate energy intake and maintain the morphology and function of intestine and intestinal epithelial cells (Tan et al., 2014; Ye et al., 2021). Moreover, Ruminiclostridium, as an intestinal beneficial bacterium, is involved in the positive regulation of the growth performance and decrease of gastrointestinal diseases of animals (Tan et al., 2014). These produced metabolites play an important role in improving intestinal environment and maintaining intestinal health. Given this phenomenon, we speculated that those genera seem to participate as key factors in maintaining the balance of gut microbiota and modulating intestinal physiological activities to further prevent diarrhea. The higher abundances of Streptococcus, Moryella, Klebsiella, Comamonas, Oscillibacter, and Rothia in the gut microbiota were closely related to many diseases including septicemia, bacteremia, endocarditis, pneumonia, and cellulitis (Boudewijns et al., 2003; Fendukly et al., 2003; Broutin et al., 2020; Kjaer et al., 2020; Liu X. J. et al., 2020; Palacio et al., 2020). Veillonellaceae may promote the development of inflammation and its abundance increase significantly in patients with inflammatory bowel disease and irritable bowel syndrome (Boudewijns et al., 2003; Fendukly et al., 2003; Broutin et al., 2020; Kjaer et al., 2020; Liu Z. et al., 2020; Palacio et al., 2020). Klebsiella, a gram-negative pathogenic bacterium, mainly distributed in the respiratory tract and intestine, which may cause pneumonia, hysteritis, mastitis, and other suppurative inflammation (Nordmann et al., 2009; Qin et al., 2020). Enterococcus has been shown to result in life-threatening meningitis, endocarditis, and sepsis (Mohanty et al., 2005). Moreover, many antibiotics frequently used in the clinic failed treating Enterococcus infection because of the inherent and acquired drug resistance (Arias and Murray, 2012). Catenibacterium was closely related to morbid obesity and metabolic syndrome (Perler et al., 2021). The relative abundance of Atopobium was significantly increased in the patients with esophageal cancer (Deng et al., 2021). Corynebacterium can result in lung abscess and caseous lymphadenitis, which was widespread in small ruminant populations (Samies et al., 1986; Dorella et al., 2006). Terrisporobacter, an emerging anaerobic pathogen, can cause surgical site infection (Cheng et al., 2016).

Gut fungal community also plays important roles in intestinal function and host health, which are in line with gut bacterial community (Paterson et al., 2017). Interestingly, although the differences in gut fungal diversity between both groups were not significant, the proportion of some intestinal fungus was altered. We observed that the diarrheic giraffes displayed increased Microascus, Aspergillus, Rhodotorula, Scopulariopsis, and Roussoella and decreased Itersonilia, Neoascochyta, Mucor, Ustilago, Protostropharia, Cephaliophora, and Xeromyces as compared with healthy populations. Microascus has been demonstrated to cause life-threatening brain abscess and pneumonia (Baddley et al., 2000; Mohammedi et al., 2004; Ustun et al., 2006; Paterson et al., 2017). Aspergillus was closely related to multiple respiratory disease, while Rhodotorula can cause fungemia (Zaas et al., 2003; Viegas et al., 2021). Scopulariopsis is characterized by its inherent resistance to the available antifungal drugs and could result in pulmonary and disseminated infections (Zaas et al., 2003; Kammoun et al., 2018; Viegas et al., 2021). Roussoella is a novel opportunistic pathogen, which can cause subcutaneous mycoses (Ahmed et al., 2014; Vasant et al., 2017). Diarrheic giraffes with altered gut microbiota may also be accompanied by weakened immunity and disease resistance. Consequently, some opportunistic pathogens may 
also display pathogenicity, which may worsen the condition or cause other diseases.

Microbes inhabiting in the gastrointestinal tract such as bacteria and fungus can form a stabilized ecosystem that plays vital roles in disease prevention, pathogenic growth inhibition, and gastrointestinal homeostasis (Ahmed et al., 2014; Hu et al., 2017; Vasant et al., 2017). Generally, the intestinal bacteria and fungi can interact in a commensal, symbiotic, or antagonistic relationship, causing a stabilized ecosystem (Aziz et al., 2013; Dabke et al., 2019). The damaged stable state of gut microbial community was regarded as the pathological mediators of multiple diseases (Sircana et al., 2018). Therefore, the altered intestinal bacteria and fungi may affect other bacterial and fungal functions, which further affect the overall intestinal functions and aggravate the gut microbial alternations (Lozupone et al., 2012). We observed that the Ruminococcaceae_UCG-014, Rikenellaceae_RC9_gut_group, and Alistipes were dramatically affected by diarrhea, and there is a significant positive correlation among them, which implied that their functions could be further affected. In the fungal community, some pathogenic bacteria such as Microascus, Aspergillus, and Rhodotorula were also significantly affected by diarrhea, and those fungi also displayed a strong positive correlation. Therefore, these pathogenic bacteria may interact with each other, which further increase their pathogenicity. This study conveyed a crucial message that diarrhea not only directly changed the gut microbial diversity and abundance but also indirectly affected other functional bacteria and fungus, which may affect intestinal functions.

\section{CONCLUSION}

In summary, this study characterized the dynamic alternations of gut bacterial and fungal communities during diarrhea in

\section{REFERENCES}

Ahmed, S. A., Stevens, D. A., van de Sande, W. W., Meis, J. F., and de Hoog, G. S. (2014). Roussoella percutanea, a novel opportunistic pathogen causing subcutaneous mycoses. Med. Mycol. 52, 689-698. doi: 10.1093/mmy/myu035

AlZahal, O., Valdes, E. V., and McBride, B. W. (2016). Analysis of the distal gut bacterial community by 454-pyrosequencing in captive giraffes (Giraffa camelopardalis). Zoo Biol. 35, 42-50. doi: 10.1002/zoo.21252

Ambalam, P., Raman, M., Purama, R. K., and Doble, M. (2016). Probiotics, prebiotics and colorectal cancer prevention. Best Pract. Res. Clin. Gastroenterol. 30, 119-131. doi: 10.1016/j.bpg.2016.02.009

Arias, C. A., and Murray, B. E. (2012). The rise of the enterococcus: beyond vancomycin resistance. Nat. Rev. Microbiol. 10, 266-278. doi: 10.1038/ nrmicro2761

Arumugam, M., Raes, J., Pelletier, E., Le Paslier, D., Yamada, T., Mende, D. R., et al. (2011). Enterotypes of the human gut microbiome. Nature 473, 174-180. doi: 10.1038/nature09944

Aziz, Q., Dore, J., Emmanuel, A., Guarner, F., and Quigley, E. M. (2013). Gut microbiota and gastrointestinal health: current concepts and future directions. Neurogastroenterol. Motil. 25, 4-15. doi: 10.1111/nmo.12046

Backhed, F., Roswall, J., Peng, Y., Feng, Q., Jia, H., Kovatcheva-Datchary, P., et al. (2015). Dynamics and stabilization of the human gut microbiome during the first year of life. Cell Host Microbe 17, 690-703. doi: 10.1016/j.chom.2015. 04.004 giraffes. Results demonstrated that the gut bacterial community in diarrheal giraffe undergoes significant changes, characterized by a decreased gut bacterial diversity and altered gut bacterial composition. Moreover, although diarrhea did not change the gut fungal diversity of giraffe, the types and proportions of some fungus have changed significantly. These results also enriched the knowledge of the gut bacterial and fungal in giraffe and convey an important message that the altered gut bacterial and fungal community may be one of the causes for the occurrence or aggravation of diarrhea. This study also provides a theoretical basis for alleviating diarrhea from the gut microbial perspective.

\section{DATA AVAILABILITY STATEMENT}

The datasets presented in this study can be found in online repositories. The names of the repository/repositories and accession number(s) can be found below: https://www.ncbi.nlm. nih.gov/, PRJNA727450.

\section{ETHICS STATEMENT}

The animal study was reviewed and approved by the Animal Welfare and Ethics Committee of Hubei Three Gorges Polytechnic.

\section{AUTHOR CONTRIBUTIONS}

$\mathrm{XJ}$ and $\mathrm{AL}$ provided the research idea. YW, BL, YF, YH, $\mathrm{HL}, \mathrm{FL}, \mathrm{HZ}$, and LW contributed to reagents, materials, and analysis tools. AL wrote the manuscript. MF-e-AK and AL revised the manuscript. All authors participated in writing and reviewing the manuscript.

Baddley, J. W., Moser, S. A., Sutton, D. A., and Pappas, P. G. (2000). Microascus cinereus (Anamorph scopulariopsis) brain abscess in a bone marrow transplant recipient. J. Clin. Microbiol. 38, 395-397. doi: 10.1128/jcm.38.1.395-397.2000

Barash, N. R., Maloney, J. G., Singer, S. M., and Dawson, S. C. (2017). Giardia alters commensal microbial diversity throughout the murine gut. Infect. Immun. 85:e0948-16. doi: 10.1128/IAI.00948-16

Boudewijns, M., Magerman, K., Verhaegen, J., Debrock, G., Peetermans, W. E., Donkersloot, P., et al. (2003). Rothia dentocariosa, endocarditis and mycotic aneurysms: case report and review of the literature. Clin. Microbiol. Infect. 9, 222-229. doi: 10.1046/j.1469-0691.2003.00503.x

Broutin, L., Deroche, L., Michaud, A., Le Moal, G., Burucoa, C., Gayet, L. E., et al. (2020). First description of bacteremia caused by Oscillibacter valericigenes in a patient hospitalized for leg amputation. Anaerobe 64:102244. doi: 10.1016/j. anaerobe.2020.102244

Brown, D. M., Brenneman, R. A., Koepfli, K. P., Pollinger, J. P., Mila, B., Georgiadis, N. J., et al. (2007). Extensive population genetic structure in the giraffe. BMC Biol. 5:57. doi: 10.1186/1741-7007-5-57

Bu, D., Zhang, X., Ma, L., Park, T., Wang, L., Wang, M., et al. (2020). Repeated inoculation of young calves with rumen microbiota does not significantly modulate the rumen prokaryotic microbiota consistently but decreases diarrhea. Front. Microbiol. 11:1403. doi: 10.3389/fmicb.2020.01403

Cai, S., Li, J., Hu, F. Z., Zhang, K., Luo, Y., Janto, B., et al. (2010). Cellulosilyticum ruminicola, a newly described rumen bacterium that possesses redundant fibrolytic-protein-encoding genes and degrades lignocellulose with multiple 
carbohydrate- borne fibrolytic enzymes. Appl. Environ. Microbiol. 76, 38183824. doi: 10.1128/AEM.03124-09

Cheng, M. P., Domingo, M. C., Levesque, S., and Yansouni, C. P. (2016). A case report of a deep surgical site infection with Terrisporobacter glycolicus/T. Mayombei and review of the literature. BMC Infect. Dis 16:529. doi: 10.1186/s12879-016-1865-8

Cryan, J. F., and Dinan, T. G. (2012). Mind-altering microorganisms: the impact of the gut microbiota on brain and behaviour. Nat. Rev. Neurosci. 13, 701-712. doi: $10.1038 /$ nrn3346

Dabke, K., Hendrick, G., and Devkota, S. (2019). The gut microbiome and metabolic syndrome. J. Clin. Invest. 129, 4050-4057. doi: 10.1172/JCI129 194

De Filippo, C., Cavalieri, D., Di Paola, M., Ramazzotti, M., Poullet, J. B., Massart, S., et al. (2010). Impact of diet in shaping gut microbiota revealed by a comparative study in children from Europe and rural Africa. Proc. Natl. Acad. Sci. U.S.A. 107, 14691-14696. doi: 10.1073/pnas.1005963107

Delzenne, N. M., Neyrinck, A. M., and Cani, P. D. (2011). Modulation of the gut microbiota by nutrients with prebiotic properties: consequences for host health in the context of obesity and metabolic syndrome. Microb. Cell Fact. 10 Suppl 1:S10. doi: 10.1186/1475-2859-10-S1-S10

Deng, Y., Tang, D., Hou, P., Shen, W., Li, H., Wang, T., et al. (2021). Dysbiosis of gut microbiota in patients with esophageal cancer. Microb. Pathog. 150:104709. doi: 10.1016/j.micpath.2020.104709

Dorella, F. A., Pacheco, L. G., Oliveira, S. C., Miyoshi, A., and Azevedo, V. (2006). Corynebacterium pseudotuberculosis: Microbiology, biochemical properties, pathogenesis and molecular studies of virulence. Vet. Res. 37, 201-218. doi: 10.1051/vetres:2005056

Downes, J., Dewhirst, F. E., Tanner, A., and Wade, W. G. (2013). Description of Alloprevotella rava gen. Nov., Sp. Nov., Isolated from the human oral cavity, and reclassification of Prevotella tannerae Moore et al. 1994 as Alloprevotella tannerae gen. Nov., Comb. Nov. Int. J. Syst. Evol. Microbiol. 63, 1214-1218. doi: 10.1099/ijs.0.041376-0

Dubin, K., Callahan, M. K., Ren, B., Khanin, R., Viale, A., Ling, L., et al. (2016). Intestinal microbiome analyses identify melanoma patients at risk for checkpoint-blockade-induced colitis. Nat. Commun. 7:10391. doi: 10.1038/ ncomms10391

Dumas, M. E., Barton, R. H., Toye, A., Cloarec, O., Blancher, C., Rothwell, A., et al. (2006). Metabolic profiling reveals a contribution of gut microbiota to fatty liver phenotype in insulin-resistant mice. Proc. Natl. Acad. Sci. U.S.A. 103, 12511-12516. doi: 10.1073/pnas.0601056103

Fendukly, F., Karlsson, I., Hanson, H. S., Kronvall, G., and Dornbusch, K. (2003). Patterns of mutations in target genes in septicemia isolates of Escherichia coli and Klebsiella pneumoniae with resistance or reduced susceptibility to ciprofloxacin. Apmis 111, 857-866. doi: 10.1034/j.1600-0463.2003.1110904.x

Gallardo, P., Izquierdo, M., Vidal, R. M., Soto, F., Ossa, J. C., and Farfan, M. J. (2020). Gut Microbiota-Metabolome changes in children with diarrhea by diarrheagenic E. coli. Front. Cell Infect. Microbiol. 10: 485. doi: 10.3389/fcimb. 2020.00485

Garrett, W. S., Gordon, J. I., and Glimcher, L. H. (2010). Homeostasis and inflammation in the intestine. Cell 140, 859-870. doi: 10.1016/j.cell.2010.01.023

Guo, W., Li, Y., Wang, L., Wang, J., Xu, Q., Yan, T., et al. (2015). Evaluation of composition and individual variability of rumen microbiota in yaks by $16 \mathrm{~S}$ rRNA high-throughput sequencing technology. Anaerobe 34, 74-79. doi: 10. 1016/j.anaerobe.2015.04.010

Han, Z., Li, K., Shahzad, M., Zhang, H., Luo, H., Qiu, G., et al. (2017). Analysis of the intestinal microbial community in healthy and diarrheal perinatal yaks by high-throughput sequencing. Microb. Pathog. 111, 60-70. doi: 10.1016/j. micpath.2017.08.025

He, B., Nohara, K., Ajami, N. J., Michalek, R. D., Tian, X., Wong, M., et al. (2015). Transmissible microbial and metabolomic remodeling by soluble dietary fiber improves metabolic homeostasis. Sci. Rep. 5:10604. doi: 10.1038/srep10604

He, K., Yan, W., Sun, C., Liu, J., Bai, R., Wang, T., et al. (2020). Alterations in the diversity and composition of gut microbiota in weaned piglets infected with Balantioides coli. Vet. Parasitol. 288:109298. doi: 10.1016/j.vetpar.2020.109298

Hong, G., Li, Y., Yang, M., Li, G., Qian, W., Xiong, H., et al. (2020). Gut fungal dysbiosis and altered bacterial-fungal interaction in patients with diarrhea-predominant irritable bowel syndrome: an explorative study. Neurogastroenterol. Motil. 32:e13891. doi: 10.1111/nmo.13891
Hu, L., Geng, S., Li, Y., Cheng, S., Fu, X., Yue, X., et al. (2017). Exogenous fecal microbiota transplantation from local adult pigs to crossbred newborn piglets. Front. Microbiol. 8:2663. doi: 10.3389/fmicb.2017.02663

Huang, C., Song, P., Fan, P., Hou, C., Thacker, P., and Ma, X. (2015). Dietary sodium butyrate decreases postweaning diarrhea by modulating intestinal permeability and changing the bacterial communities in weaned piglets. J. Nutr. 145, 2774-2780. doi: 10.3945/jn.115.217406

Kammoun, S., Rekik, M., Trabelsi, H., Neji, S., Feki, J., and Ayadi, A. (2018). Orbital cellulitis secondary to a fungal sinusitis caused by Scopulariopsis: the first case in Tunisia. J. Mycol. Med. 28, 384-386. doi: 10.1016/j.mycmed.2018.04.006

Kjaer, H. S., Lofberg, S. V., Nielsen, D. K., Kobbero, H., and Justesen, U. S. (2020). Bacteraemia with moryella indoligenes and Fastidiosipila sanguinis: a case report. Access Microbiol. 2:acmi000108. doi: 10.1099/acmi.0.000108

Li, A., Wang, Y., He, Y., Liu, B., Iqbal, M., Mehmood, K., et al. (2021a). Environmental fluoride exposure disrupts the intestinal structure and gut microbial composition in ducks. Chemosphere 277:130222. doi: 10.1016/j. chemosphere.2021.130222

Li, A., Yang, Y., Zhang, Y., Lv, S., Jin, T., Li, K., et al. (2021b). Microbiome analysis reveals the alterations in gut microbiota in different intestinal segments of Yimeng black goats. Microb. Pathog. 155:104900. doi: 10.1016/j.micpath.2021. 104900

Li, K., Mehmood, K., Zhang, H., Jiang, X., Shahzad, M., Dong, X., et al. (2018). Characterization of fungus microbial diversity in healthy and diarrheal yaks in Gannan region of Tibet Autonomous Prefecture. Acta Trop. 182, 14-26. doi: 10.1016/j.actatropica.2018.02.017

Liu, X. J., Qiao, X. W., Huang, T. M., Li, L., and Jiang, S. P. (2020). Comamonas kerstersii bacteremia. Med. Mal. Infect. 50, 288-290. doi: 10.1016/j.medmal. 2019.12.005

Liu, Z., Li, A., Wang, Y., Iqbal, M., Zheng, A., Zhao, M., et al. (2020). Comparative analysis of microbial community structure between healthy and Aeromonas veronii-infected Yangtze finless porpoise. Microb. Cell Fact. 19:123. doi: 10. 1186/s12934-020-01383-4

Lozupone, C. A., Stombaugh, J. I., Gordon, J. I., Jansson, J. K., and Knight, R. (2012). Diversity, stability and resilience of the human gut microbiota. Nature 489, 220-230. doi: 10.1038/nature11550

Ma, Z., Wang, H.-J., Ma, X.-J., Li, Y., and Yang, H.-J., Li, H., et al. (2020). Modulation of gut microbiota and intestinal barrier function during alleviation of antibiotic-associated diarrhea with Rhizoma Zingiber officinale (Ginger) extract. Food Funct. 11, 10839-10851. doi: 10.1039/d0fo01536a

Manichanh, C., Borruel, N., Casellas, F., and Guarner, F. (2012). The gut microbiota in IBD. Nat. Rev. Gastroenterol. Hepatol. 9, 599-608. doi: 10.1038/nrgastro. 2012.152

McCracken, B. A., and Nathalia, G. M. (2020). Phylum Synergistetes in the oral cavity: a possible contributor to periodontal disease. Anaerobe 68:102250. doi: 10.1016/j.anaerobe.2020.102250

Miao, V., and Davies, J. (2010). Actinobacteria: the good, the bad, and the ugly. Antonie Van Leeuwenhoek 98, 143-150. doi: 10.1007/s10482-010-9440-6

Mohammedi, I., Piens, M. A., Audigier-Valette, C., Gantier, J. C., Argaud, L., Martin, O., et al. (2004). Fatal Microascus trigonosporus (anamorph Scopulariopsis) pneumonia in a bone marrow transplant recipient. Eur. J. Clin. Microbiol. Infect. Dis. 23, 215-217. doi: 10.1007/s10096-003-1096-y

Mohanty, S., Dhawan, B., Kapil, A., Das, B. K., Pandey, P., and Gupta, A. (2005). Brain abscess due to Enterococcus avium. Am. J. Med. Sci. 329, 161-162. doi: 10.1097/00000441-200503000-00011

Mulherin, E., Bryan, J., Beltman, M., O’Grady, L., Pidgeon, E., Garon, L., et al. (2008). Molecular characterisation of a bovine-like rotavirus detected from a giraffe. BMC Vet. Res. 4:46. doi: 10.1186/1746-6148-4-46

Musso, G., Gambino, R., and Cassader, M. (2011). Interactions between gut microbiota and host metabolism predisposing to obesity and diabetes. Annu. Rev. Med. 62, 361-380. doi: 10.1146/annurev-med-012510-175505

Nordmann, P., Cuzon, G., and Naas, T. (2009). The real threat of Klebsiella pneumoniae carbapenemase-producing bacteria. Lancet Infect. Dis. 9, 228-236. doi: 10.1016/S1473-3099(09)70054-4

O’Keefe, S. J., Li, J. V., Lahti, L., Ou, J., Carbonero, F., Mohammed, K., et al. (2015). Fat, fibre and cancer risk in african americans and rural africans. Nat. Commun. 6:6342. doi: $10.1038 /$ ncomms 7342

Palacio, R., Cabezas, L., Cornejo, C., and Seija, V. (2020). [Comamonas kerstersii bacteremia in a young man with acute appendicitis]. Rev. 
Chilena. Infectol. 37, 182-185. doi: 10.4067/s0716-101820200002 00182

Paterson, M. J., Oh, S., and Underhill, D. M. (2017). Host-microbe interactions: commensal fungi in the gut. Curr. Opin. Microbiol. 40, 131-137. doi: 10.1016/j. mib.2017.11.012

Peng, B., Huang, S., Liu, T., and Geng, A. (2015). Bacterial xylose isomerases from the mammal gut Bacteroidetes cluster function in Saccharomyces cerevisiae for effective xylose fermentation. Microb. Cell Fact. 14:70. doi: 10.1186/s12934-0150253-1

Perler, B. K., Reinhart, E. M., Montgomery, M., Maynard, M., Shapiro, J. M., Belenky, P., et al. (2021). Evaluation of the microbiome in men taking preexposure prophylaxis for HIV prevention. AIDS Behav. 25, 2005-2013. doi: 10.1007/s10461-020-03130-7

Qin, X., Wu, S., Hao, M., Zhu, J., Ding, B., Yang, Y., et al. (2020). The colonization of carbapenem-resistant klebsiella pneumoniae: epidemiology, resistance mechanisms, and risk factors in patients admitted to intensive care units in china. J. Infect. Dis. 221, S206-S214. doi: 10.1093/infdis/jiz622

Ricaboni, D., Mailhe, M., Khelaifia, S., Raoult, D., and Million, M. (2016). Romboutsia timonensis, a new species isolated from human gut. New Microbes New Infect. 12, 6-7. doi: 10.1016/j.nmni.2016.04.001

Samies, J. H., Hathaway, B. N., Echols, R. M., Veazey, J. J., and Pilon, V. A. (1986). Lung abscess due to Corynebacterium equi. Report of the first case in a patient with acquired immune deficiency syndrome. Am. J. Med. 80, 685-688. doi: 10.1016/0002-9343(86)90825-9

Sangster, W., Hegarty, J. P., Schieffer, K. M., Wright, J. R., Hackman, J., Toole, D. R., et al. (2016). Bacterial and fungal microbiota changes distinguish C. Difficile infection from other forms of diarrhea: results of a prospective inpatient study. Front. Microbiol. 7:789. doi: 10.3389/fmicb.2016.00789

Shang, Q., Shan, X., Cai, C., Hao, J., Li, G., and Yu, G. (2016). Dietary fucoidan modulates the gut microbiota in mice by increasing the abundance of Lactobacillus and Ruminococcaceae. Food Funct. 7, 3224-3232. doi: 10.1039/ c6fo00309e

Shao, H., Zhang, C., Xiao, N., and Tan, Z. (2020). Gut microbiota characteristics in mice with antibiotic-associated diarrhea. BMC Microbiol. 20:313. doi: 10.1186/ s12866-020-01999-x

Shukla, R., Ghoshal, U., Dhole, T. N., and Ghoshal, U. C. (2015). Fecal microbiota in patients with irritable bowel syndrome compared with healthy controls using Real-Time polymerase chain reaction: an evidence of dysbiosis. Dig. Dis. Sci. 60, 2953-2962. doi: 10.1007/s10620-015-3607-y

Sircana, A., Framarin, L., Leone, N., Berrutti, M., Castellino, F., Parente, R., et al. (2018). Altered gut microbiota in type 2 diabetes: just a coincidence? Curr. Diab. Rep. 18:98. doi: 10.1007/s11892-018-1057-6

Sun, B., Wang, X., Bernstein, S., Huffman, M. A., Xia, D. P., Gu, Z., et al. (2016). Marked variation between winter and spring gut microbiota in freeranging Tibetan Macaques (Macaca thibetana). Sci. Rep. 6:26035. doi: 10.1038/ srep 26035

Tan, J., McKenzie, C., Potamitis, M., Thorburn, A. N., Mackay, C. R., and Macia, L. (2014). The role of short-chain fatty acids in health and disease. Adv. Immunol. 121, 91-119. doi: 10.1016/B978-0-12-800100-4.00003-9

Tremaroli, V., and Backhed, F. (2012). Functional interactions between the gut microbiota and host metabolism. Nature 489, 242-249. doi: 10.1038/ nature11552

Ustun, C., Huls, G., Stewart, M., and Marr, K. A. (2006). Resistant Microascus cirrosus pneumonia can be treated with a combination of surgery, multiple antifungal agents and a growth factor. Mycopathologia 162, 299-302. doi: 10.1007/ s11046-006-0067-0

Vartoukian, S. R., Palmer, R. M., and Wade, W. G. (2009). Diversity and morphology of members of the phylum "synergistetes" in periodontal health and disease. Appl. Environ. Microbiol. 75, 3777-3786. doi: 10.1128/AEM.02 763-08

Vasant, J. A., Maggiani, F., and Borman, A. M. (2017). Subcutaneous mycotic cyst caused by roussoella percutanea in a UK renal transplant patient. Mycopathologia 182, 721-725. doi: 10.1007/s11046-017-0121-0

Viegas, C., Caetano, L. A., and Viegas, S. (2021). Occupational exposure to Aspergillus section Fumigati: tackling the knowledge gap in Portugal. Environ Res 194, 110674. doi: 10.1016/j.envres.2020.110674
Wang, J., Zhu, G., Sun, C., Xiong, K., Yao, T., Su, Y., et al. (2020). TAK-242 ameliorates DSS-induced colitis by regulating the gut microbiota and the JAK2/STAT3 signaling pathway. Microb. Cell Fact. 19:158. doi: 10.1186/s12934$020-01417-x$

Wang, Y., Li, A., Liu, J., Mehmood, K., Wangdui, B., Shi, H., et al. (2019a). L. Pseudomesenteroides and L. Johnsonii isolated from yaks in Tibet modulate gut microbiota in mice to ameliorate enteroinvasive Escherichia coli-induced diarrhea. Microb. Pathog. 132, 1-9. doi: 10.1016/j.micpath.2019.04.020

Wang, Y., Li, A., Zhang, L., Waqas, M., Mehmood, K., Iqbal, M., et al. (2019b). Probiotic potential of Lactobacillus on the intestinal microflora against Escherichia coli induced mice model through high-throughput sequencing. Microb. Pathog. 137:103760. doi: 10.1016/j.micpath.2019.103760

Wang, Y., Zhang, H., Zhu, L., Xu, Y., Liu, N., Sun, X., et al. (2018). Dynamic distribution of gut microbiota in goats at different ages and health states. Front. Microbiol. 9:2509. doi: 10.3389/fmicb.2018.02509

Xiang, L., Wu, Q., Osada, H., Yoshida, M., Pan, W., and Qi, J. (2020). Peanut skin extract ameliorates the symptoms of type 2 diabetes mellitus in mice by alleviating inflammation and maintaining gut microbiota homeostasis. Aging (Albany NY) 12, 13991-14018. doi: 10.18632/aging.103521

Xin, J., Chai, Z., Zhang, C., Zhang, Q., Zhu, Y., Cao, H., et al. (2019). Comparing the microbial community in four stomach of dairy cattle, yellow cattle and three yak herds in Qinghai-Tibetan plateau. Front. Microbiol. 10:1547. doi: 10.3389/fmicb.2019.01547

Xiong, L., You, J., Zhang, W., Zhu, Q., Blachier, F., Yin, Y., et al. (2020). Intrauterine growth restriction alters growth performance, plasma hormones, and small intestinal microbial communities in growing-finishing pigs. J. Anim. Sci. Biotechnol. 11:86. doi: 10.1186/s40104-020-00490-x

Xue, N. Y., Liu, F., Tao, W. F., Zhao, Q., Qiu, H. Y., Hu, Y., et al. (2020). Molecular detection of Cryptosporidium spp. And Enterocytozoon bieneusi in longjiang wagyu cattle in Northeastern China. Microb. Pathog. 149:104526. doi: 10.1016/ j.micpath.2020.104526

Yang, Q., Huang, X., Zhao, S., Sun, W., Yan, Z., Wang, P., et al. (2017). Structure and function of the fecal microbiota in diarrheic neonatal piglets. Front. Microbiol. 8:502. doi: $10.3389 /$ fmicb.2017.00502

Ye, X., Zhou, L., Zhang, Y., Xue, S., Gan, Q. F., and Fang, S. (2021). Effect of host breeds on gut microbiome and serum metabolome in meat rabbits. BMC Vet. Res. 17:24. doi: 10.1186/s12917-020-02732-6

Zaas, A. K., Boyce, M., Schell, W., Lodge, B. A., Miller, J. L., and Perfect, J. R. (2003). Risk of fungemia due to Rhodotorula and antifungal susceptibility testing of Rhodotorula isolates. J. Clin. Microbiol. 41, 5233-5235. doi: 10.1128/jcm.41.11. 5233-5235.2003

Zhang, L., Jiang, X., Li, A., Waqas, M., Gao, X., Li, K., et al. (2020). Characterization of the microbial community structure in intestinal segments of yak (Bos grunniens). Anaerobe 61:102115. doi: 10.1016/j.anaerobe.2019. 102115

Zhu, L., Xu, F., Wan, W., Yu, B., Tang, L., Yang, Y., et al. (2020). Gut microbial characteristics of adult patients with allergy rhinitis. Microb. Cell Fact. 19:171. doi: 10.1186/s12934-020-01430-0

Conflict of Interest: The authors declare that the research was conducted in the absence of any commercial or financial relationships that could be construed as a potential conflict of interest.

Publisher's Note: All claims expressed in this article are solely those of the authors and do not necessarily represent those of their affiliated organizations, or those of the publisher, the editors and the reviewers. Any product that may be evaluated in this article, or claim that may be made by its manufacturer, is not guaranteed or endorsed by the publisher.

Copyright ( 2021 Li, Liu, Li, He, Wang, Fakhar-e-Alam Kulyar, Li, Fu, Zhu, Wang and Jiang. This is an open-access article distributed under the terms of the Creative Commons Attribution License (CC BY). The use, distribution or reproduction in other forums is permitted, provided the original author(s) and the copyright owner(s) are credited and that the original publication in this journal is cited, in accordance with accepted academic practice. No use, distribution or reproduction is permitted which does not comply with these terms. 